\title{
The last pieces of the primitive inner belt puzzle: Klio, Chaldaea, Chimaera, and Svea ${ }^{\star}$
}

\author{
David Morate ${ }^{1}$, Julia de León ${ }^{2,3}$, Mário De Prá ${ }^{4}$, Javier Licandro ${ }^{2,3}$, Noemí Pinilla-Alonso ${ }^{4,5}$, Humberto Campins ${ }^{6}$, \\ Anicia Arredondo ${ }^{6}$, Jorge Marcio Carvano ${ }^{1}$, Daniela Lazzaro ${ }^{1}$, and Antonio Cabrera-Lavers ${ }^{2,7}$ \\ ${ }^{1}$ Observatório Nacional, Coordenação de Astronomia e Astrofísica, 20921-400 Rio de Janeiro, Brazil \\ e-mail: davidmorate@on.br \\ 2 Instituto de Astrofísica de Canarias (IAC), C/Vía Láctea s/n, 38205 La Laguna, Tenerife, Spain \\ 3 Departamento de Astrofísica, Universidad de La Laguna, 38205 La Laguna, Tenerife, Spain \\ ${ }^{4}$ Florida Space Institute, University of Central Florida, Orlando, FL 32816, USA \\ 5 Arecibo Observatory, University of Central Florida, HC-3 Box 53995, Arecibo 00612, Puerto Rico \\ ${ }^{6}$ Physics Department, University of Central Florida, PO Box 162385, Orlando, FL 32816-2385, USA \\ ${ }^{7}$ GTC Project Office, 38205 La Laguna, Tenerife, Spain
}

Received 30 May 2019 / Accepted 16 July 2019

\begin{abstract}
Aims. Several primitive families in the inner region of the main asteroid belt were identified as potential sources for two near-Earth asteroids (NEAs), (101955) Bennu and (162173) Ryugu, targets of the sample-return missions OSIRIS-REx and Hayabusa2, respectively. Four of the families, located at high proper inclinations $\left(i>10^{\circ}\right)$, have not yet been compositionally studied: Klio, Chaldaea, Chimaera, and Svea. We want to characterize and analyze these families within the context of our PRIMitive Asteroid Spectroscopic Survey (PRIMASS), in order to complete the puzzle of the origins of the two NEAs.

Methods. We obtained visible spectra $(0.5-0.9 \mu \mathrm{m})$ of a total of 73 asteroids within the Klio, Chaldaea, Chimaera, and Svea collisional families, using the instrument OSIRIS at the $10.4 \mathrm{~m}$ Gran Telescopio Canarias. We performed a taxonomical classification of these objects, and an analysis of the possible presence of absorption bands related to aqueous alterations, comparing the results with already studied primitive families in the inner main belt.

Results. We present here reflectance spectra for 30 asteroids in the Klio family, 15 in Chaldaea, 20 in Chimaera, and 8 in Svea. We show that Klio, Chaldaea, and Chimaera members have moderately red spectral slopes, with aqueous alteration absorption bands centered around $0.7 \mu \mathrm{m}$, characteristic of the group of primitive families known as Erigone-like. In contrast, Svea shows no $0.7 \mu \mathrm{m}$ features, and neutral and blue spectral slopes, and thus is a Polana-like family. While all four families might be related to (162173) Ryugu, the only family studied in this work that might be related to (101955) Bennu is Svea.
\end{abstract}

Key words. minor planets, asteroids: general - techniques: spectroscopic

\section{Introduction}

Asteroid families are the leftovers of catastrophic collisional events in the Solar System. After such episodes, fragments of a larger body that share orbital and compositional properties are left behind (also referred to as family members). These fragments then undergo dynamical evolution, mainly through the Yarkovsky effect, gradually reaching their current locations in the main belt (Opik 1951; Bottke et al. 2002a; Vokrouhlický et al. 2006). This effect, combined with the presence of orbital resonances, creates transport routes to deliver asteroids to the nearEarth region. Since the orbits of near-Earth asteroids (NEAs) have short lifetimes compared with the age of the Solar System (Morbidelli et al. 2002), it is thought that these objects originated somewhere else and were then transported to their current orbits by a replenishment mechanism.

The inner part of the main asteroid belt, bounded by two major resonances, namely the $v_{6}$ secular resonance and the 3:1 mean motion resonance, is considered the principal source of NEAs (Bottke et al. 2002b). These objects have, on average,

\footnotetext{
* A copy of the spectra is available at the CDS via anonymous ftp to cdsarc.u-strasbg. fr (130.79.128.5) or via http://cdsarc. u-strasbg.fr/viz-bin/cat/J/A+A/630/A141
}

smaller diameters than main belt asteroids, hence unlikely to be primordial objects. Since the family forming events generate chunks of small sizes, with diameters similar to those of NEAs, collisional families are favored against isolated asteroids when looking for NEAs origins.

Determining the sources of NEAs is particularly important in the case of targets of space missions as it enhances their scientific return. In recent years, four primitive asteroid families were proposed as the most likely sources for asteroids (101955) Bennu, target of the NASA's OSIRIS-REx mission, and (162173) Ryugu, target of the JAXA's Hayabusa2 mission. All four families, the Polana-Eulalia complex, Erigone, Sulamitis, and Clarissa, are located in the inner region of the main asteroid belt at inclinations lower than $10^{\circ}$ (Campins et al. 2010, 2013; Bottke et al. 2015). These primitive families are mainly populated by carbonaceous asteroids with low albedos $\left(p_{V}<0.10\right)$ and almost featureless visible spectra; an important fraction of the objects present a shallow absorption band centered around $0.7 \mu \mathrm{m}$ in their spectrum, related to hydrated minerals on their surfaces (Vilas \& Gaffey 1989; Vilas 1994; Howell et al. 2011; Rivkin 2012).

In addition to the four families previously mentioned, another group of four primitive-candidate families was identified by 

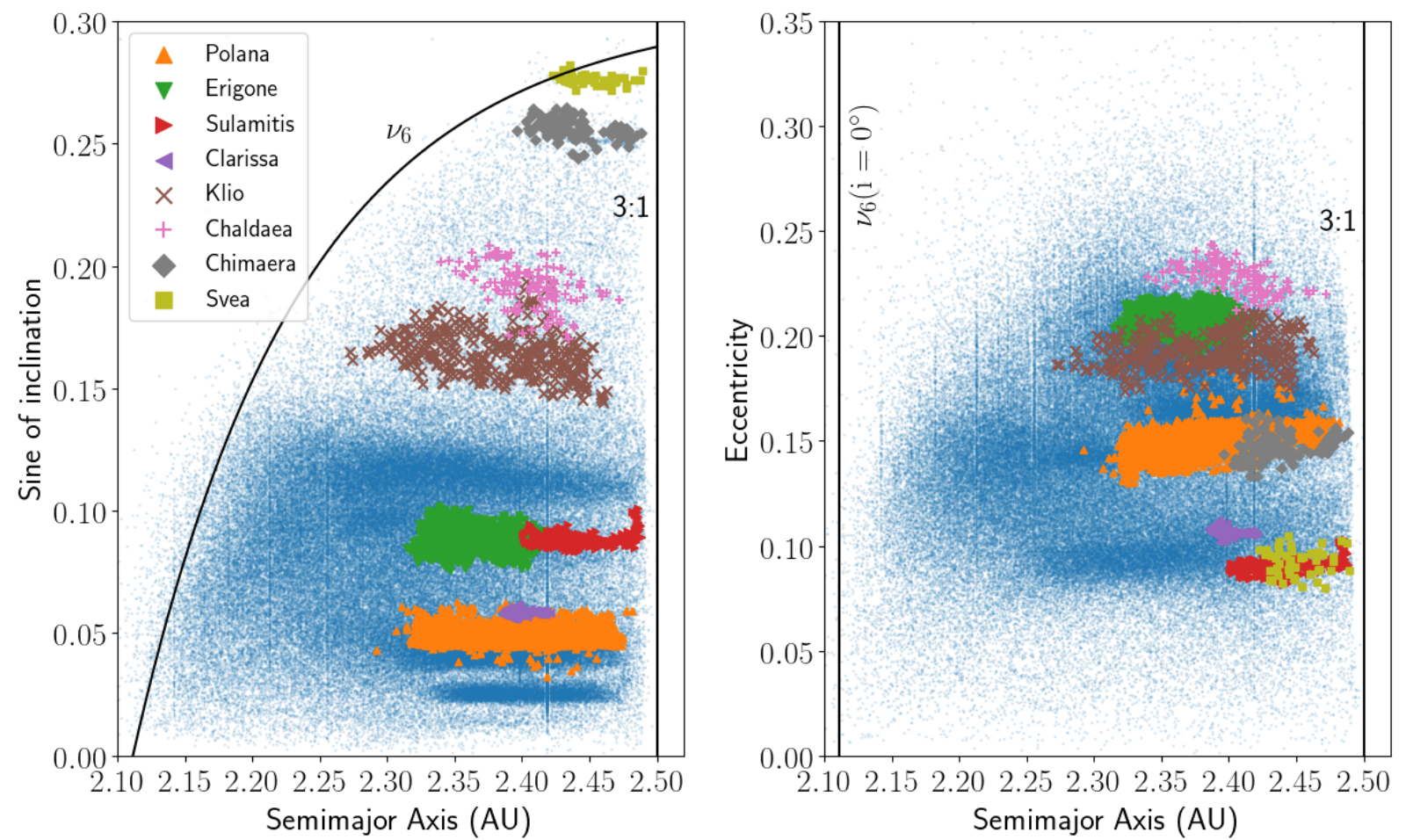

Fig. 1. Proper semimajor axis $(a)$ vs. proper sine of inclination $(l e f t)$ and proper eccentricity $(r i g h t)$ for the eight primitive families in the inner main belt. The high-inclination families studied in this work are Klio (brown crosses, $\times$ ), Chaldaea (pink plus signs, + ), Chimaera (grey diamonds, $\diamond$ ), and Svea (yellow squares, $\square$ ). The blue dots are the asteroids with computed proper elements present in the inner main belt, and the black lines limiting this region represent the $v_{6}$ and 3:1 resonances.

Nesvorný et al. (2015), with proper inclinations above $10^{\circ}$ : Klio, Chaldaea, Chimaera, and Svea (see Fig. 1). Throughout this work, we refer to this group as the high-inclination families; instead, we refer to the previously studied groups as the lowinclination families. Due to the proximity of the high-inclination families to the $v_{6}$ and 3:1 resonances, and the information provided by SDSS visible colors (Ivezić et al. 2001; Nesvorný et al. 2015), these families, although considerably smaller than their low-inclination counterparts (a total of 618 asteroids within the four families), form one last block of potential sources for primitive NEAs.

With the main goal of characterizing the possible origins of (101955) Bennu and (162173) Ryugu, in 2010 we started our PRIMitive Asteroids Spectroscopic Survey (PRIMASS), a spectroscopic survey in the visible and near-infrared with the aim of studying the primitive collisional families in the inner belt. It includes spectra from primitive families in the mid and outer belt, and other groups of primitive asteroids such as the Hildas and Cybeles. At the moment we have spectroscopic information for more than 500 asteroids.

As part of this survey we observed and characterized the four low-inclination primitive families in the inner main belt: Polana-Eulalia, Erigone, Sulamitis, and Clarissa. Results for the four families from visible spectroscopy have been published in Morate et al. (2016, 2018) and de León et al. (2016, 2018), where most of the spectra were acquired with the $10.4 \mathrm{~m}$ Gran Telescopio Canarias (GTC), located at the El Roque de los Muchachos Observatory, on the island of La Palma (Spain). The Polana-Eulalia complex has also been studied via near-infrared spectroscopy using both the Telescopio Nazionale Galileo (TNG) and the NASA Infrared Telescope Facility (see PinillaAlonso et al. 2016). As stated before, the Hilda and Cybele groups, in the outer main belt, have also been studied within PRIMASS (De Prá et al. 2018a).
To go forward with our PRIMASS survey, and to place the last pieces of the puzzle regarding the possible origins of (101955) Bennu and (162173) Ryugu, we observed and characterized the high-inclination primitive-candidate families in the inner main belt: Klio, Chaldaea, Chimaera, and Svea. These families were observed during semesters 2016B and 2017A (September 2016-August 2017), following procedures for the data acquisition, reduction, and compositional analysis similar to those described in Morate et al. (2016, 2018). The observations and data reduction are described in Sect. 2. The taxonomical classification, slope analyses, and absorption band search are presented in Sect. 3. We discuss the results of the analysis in Sect. 4, and we summarize the conclusions and discuss future work in Sect. 5.

\section{Observations and data reduction}

All the asteroids analyzed in the present study were selected from the families within the Nesvorny (2015) dataset ${ }^{1}$. This dataset contains asteroid dynamical family memberships for 122 families calculated from synthetic proper elements, including high-inclination families. These families were computed in Nesvorný et al. (2015), using a code based on the hierarchical clustering method (HCM), described in Zappala et al. (1990) and Zappala \& Cellino (1994). It is interesting to note that the four families studied here were not reported in Milani et al. (2014), the other main work in the field. This is due to the method used in their work; they remove fainter asteroids $(H>15)$ as a previous step to the family identification. Since the asteroids in the Klio, Chaldaea, Chimaera, and Svea families are mainly fainter than that threshold $(75,76,83$, and $90 \%$, respectively), it is not surprising that they are not classified as families by Milani et al. (2014).

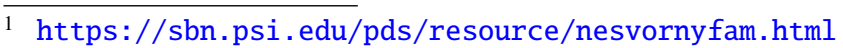


Table 1. Right ascension, declination, and visible magnitude for the solar analog stars used to obtain the reflectance spectra of the observed asteroids.

\begin{tabular}{llccc}
\hline \hline ID & Star & $\alpha$ & $\delta$ & $V$ \\
\hline 1 & SA 93-101 & $01: 53: 18$ & $+00: 22: 23$ & 9.7 \\
2 & SA 98-978 & $06: 51: 34$ & $-00: 11: 32$ & 10.6 \\
3 & SA 102-1081 & $10: 57: 04$ & $-00: 13: 13$ & 9.9 \\
4 & SA 107-998 & $15: 38: 16$ & $+00: 15: 22$ & 10.5 \\
5 & SA 110-361 & $18: 42: 45$ & $+00: 08: 05$ & 12.4 \\
6 & SA 112-1333 & $20: 43: 12$ & $+00: 26: 13$ & 9.9 \\
7 & SA 115-271 & $23: 42: 42$ & $+00: 45: 13$ & 9.7 \\
8 & Hyades 64 & $04: 26: 40$ & $+16: 44: 49$ & 8.1 \\
9 & M67-1194 & $08: 51: 01$ & $+11: 48: 53$ & 14.7 \\
\hline
\end{tabular}

Notes. The ID number is the same as that in the next to last column of Table B.1.

We used the Nesvorny (2015) dataset since it is the most recent work in asteroid families identification. According to the last version of the dataset, the Klio, Chaldaea, Chimaera, and Svea families have 330, 132, 108, and 48 members each, respectively. The proper orbital elements (and the computed absolute magnitudes) of the asteroids observed in this work can be found within this dataset. Due to the reduced number of objects in the four families, we did not impose any observational constraints. The objects to be observed were selected only according to their visibility throughout the semester.

For this work, a total of 73 low-resolution visible spectra of asteroids within the Klio (30), Chaldaea (15), Chimaera (20), and Svea (8) families were acquired using the OSIRIS $^{2}$ camera spectrograph (Cepa et al. 2000; Cepa 2010) at the $10.4 \mathrm{~m}$ Gran Telescopio Canarias (GTC) located at the El Roque de los Muchachos observatory (ORM) in La Palma, Canary Islands (Spain). The configuration of the spectrograph and the observational method were the same as those described in Morate et al. (2018). A summary of the observational circumstances of the asteroids studied in this work is shown in Table B.1. In this table we also include the solar analogue stars used to obtain the reflectance spectra of each object. The observations were done in service mode, under GTC programs GTC22-16B and GTC6817A, from September (2016) through August (2017). Since these observational programs are classified as Band $\mathrm{C}$ or fillers, the final spectra quality might vary from one night to another. This is due to the weather conditions, and to the night qualities, being variable within filler programs, including high seeing values, bright nights, or even some cirrus coverage.

Every night, in addition to the asteroids, at least one solar analog star was observed. These solar analog stars were observed with the same instrument configuration used for the asteroids, and at similar airmasses. This is done in order to correct for telluric absorptions. The asteroid reflectance spectra is obtained after removing the solar contribution (i.e., dividing the asteroid spectra by that of the star). The solar analogs that we used are shown in Table 1.

The data reduction process is done in two main steps. First, we used standard IRAF $^{3}$ tasks for image processing: bias and

\footnotetext{
2 Information about the specifications of the OSIRIS instrument can be found at http: //www.gtc.iac.es/instruments/osiris/

3 IRAF is distributed by the National Optical Astronomy Observatories, which are operated by the Association of Universities for Research in Astronomy, Inc., under cooperative agreement with the National Science Foundation.
}

flat-field correction, spectrum extraction, and wavelength calibration. Second, we used several Python routines in order to align and then divide the asteroid spectra by that of the solar ana$\log$. This routine also normalizes the spectra to unity at $0.55 \mu \mathrm{m}^{4}$. Final spectra for all the families are shown in Figs. A.1-A.4. See Morate et al. $(2016,2018)$ for a more detailed description of the reduction process.

\section{Analysis and results}

In this section we detail the analysis that we performed on the obtained spectra: taxonomic classification, slope computation, and the absorption band search. The results are shown in Table B.2.

\subsection{Taxonomy determination}

Using the spectra obtained after the reduction process, we performed a taxonomical analysis to classify the observed asteroids in order to infer the possible surface composition of these objects. For this, we used a toolkit developed by our group to facilitate the study of spectroscopic and photometric asteroid data: the Python package Codes for ANalysis of Asteroids (CANA, De Prá et al. 2018b). The method used by CANA to compute the taxonomy of an object is based on the Bus-DeMeo taxonomy (DeMeo et al. 2009), which is an extension to the near-infrared of the Bus taxonomy in the visible (Bus \& Binzel 2002a). Since the majority of the classes in the Bus-DeMeo taxonomy overlap with those from the Bus taxonomy, we considered the CANA output equivalent to the Bus taxonomy and classified the spectra according to Bus.

The CANA classification method is based on the $\chi^{2}$ analysis (Bevington \& Robinson 1992). The pipeline returns a default number of three possible classifications, along with their related chi-squared values. We selected the classification associated with the lowest chi-square value, except for the case of asteroid 7030, in which the lowest chi-square value referred to an Sv type (a class that does not exist in the Bus taxonomy), so we selected the second taxonomical class proposed for this object, which was A-type. The taxonomical classification ${ }^{5}$ obtained for each asteroid is shown in the second column of Table B.2.

This classification procedure for the four families yielded the following numbers: for the Klio family, we found a total of 4 B-types, 17 C-types, 8 X-types, and 1 D-type; for the Chaldaea family, we found $12 \mathrm{C}$-types, $2 \mathrm{X}$-types, and $1 \mathrm{~A}$-type; for the Chimaera family we found $12 \mathrm{C}$-types, $4 \mathrm{X}$-types, 1 T-type, and 3 D-types; and finally, for the Svea family, we found 4 B-types, $3 \mathrm{C}$-types, and $1 \mathrm{X}$-type. These results are summarized in the pie charts presented in Fig. 2.

We see an apparent predominance of C-types for three of these families (Klio, Chaldaea, and Chimaera), compared to the higher percentage of B-types in Svea. Although the percentage of observed objects in Svea is the highest of the four families $(\sim 16 \%)$, we have to take into account the fact that the number of observed asteroids is reduced (just eight objects).

\subsection{Spectral slope computation}

Within the full sample of asteroids that we obtained, we found that all of them, except for one object, showed the typical

\footnotetext{
4 Central wavelength of the $V$ Johnson filter, widely used as reference for the normalization of Solar System objects in the visible wavelength range.

5 Throughout this article, we refer to C-type asteroids (the group comprising C-, Cb-, Cg-, Ch-, and Cgh-type asteroids), and $\mathrm{X}$-type asteroids (the group comprising $\mathrm{X}-, \mathrm{Xc}-\mathrm{Xe}$-, and $\mathrm{Xk}$-type asteroids).
} 


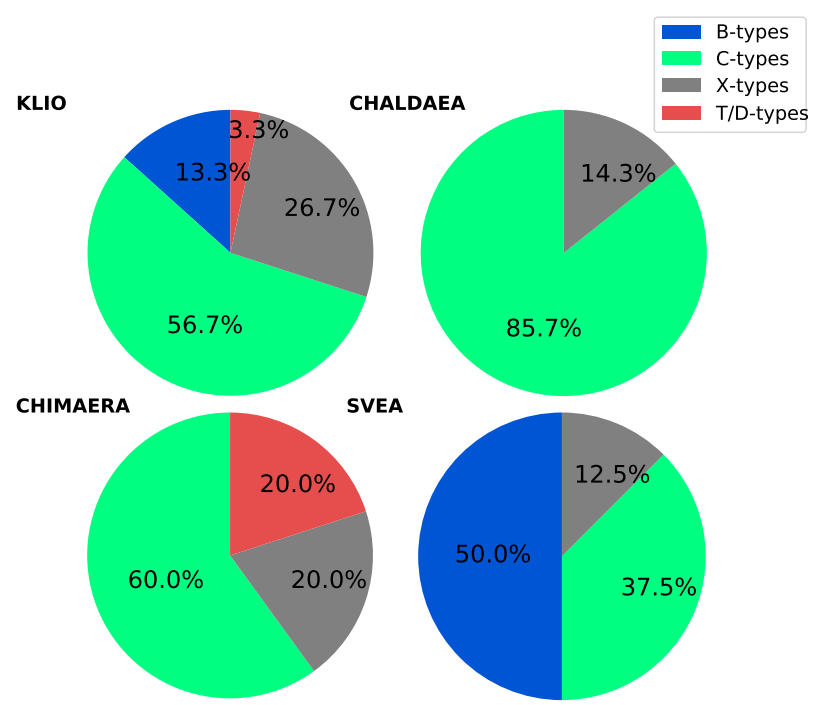

Fig. 2. Distribution of the taxonomical classes for the four families studied in this work. We have excluded from this graphical representation one A-type asteroid from the Chaldaea family (the only non-primitive asteroid found in the data).

featureless spectra related to what we know as primitive objects. For these 72 asteroids, we computed their spectral slopes. The definition that we used is the one by Luu \& Jewitt (1990). We used spectral data in the $0.55-0.90 \mu \mathrm{m}$ range to compute it. In order to compute the spectral slopes, we first fitted our spectrum in this spectral range using a first-order polynomial (i.e., fitting the spectrum to a straight line). The slope of this fit, divided by the value of its first point (i.e., the reflectance of the fit at $0.55 \mu \mathrm{m})$, is the value that we use for the spectral slope. It is measured in units of in units of percentage per $1000 \AA$.

The errors associated with the spectral slope values are given by the statistical uncertainties in the fit calculation, and also by the use of different solar analog stars (which we could consider a "systematic error" of the measurements). However, since the uncertainties associated with the linear fit are two orders of magnitude smaller than the variation in the slope due to the solar analogs ${ }^{6}$, we will consider an error of $\pm 0.9 \% / 1000 \AA$ in all cases. The resulting values for the computed spectral slopes are shown in Table B.2.

We show the different slope distributions in Fig. 3, comparing each family studied in this paper to the other three. Klio and Chaldaea present a similar slope distribution, with similar mean values: $1.16 \pm 2.04 \%$ (Klio) and $0.88 \pm 1.25 \%$ (Chaldaea)/ $1000 \AA$. Chimaera and Svea, on the other hand, show different traits: Chimaera presents redder slopes (and a mean slope value of $2.82 \pm 3.39 \% / 1000 \AA$ ), while Svea seems bluer than the other three families (with a mean slope of $-0.52 \pm 2.04 \% / 1000 \AA$ ). We discuss this further and expand the comparison to the rest of primitive families in the inner main belt in Sect. 4 .

\subsection{Aqueous alteration}

As stated in Sect. 1, the visible spectra of primitive asteroids are usually featureless, being the main spectral characteristic a shallow absorption band, centered around $0.7 \mu \mathrm{m}$ (Vilas 1994; Fornasier et al. 1999, 2014; Carvano et al. 2003; Rivkin 2012;

6 The value for this systematic error is obtained by comparing the spectra of different solar analogs on those nights where two or more solar analogs were observed. For the present study, this value is $0.9 \% /$ $1000 \AA$.
Table 2. Results from the analysis of the aqueous alteration band within the four families studied in this paper.

\begin{tabular}{lcccccc}
\hline \hline Family & $N_{\mathrm{H}}$ & $N_{\mathrm{U}}$ & $N_{\mathrm{T}}$ & $\begin{array}{c}\overline{\text { Center }} \\
(\AA)\end{array}$ & $\begin{array}{c}\overline{\text { Depth }} \\
(\%)\end{array}$ & $\%$ \\
\hline Klio & 7 & 2 & 30 & $7000 \pm 150$ & $2.2 \pm 0.6$ & 23 \\
Chaldaea & 11 & 0 & 14 & $7110 \pm 130$ & $3.1 \pm 0.8$ & 79 \\
Chimaera & 4 & 4 & 20 & $7100 \pm 150$ & $2.9 \pm 1.2$ & 20 \\
Svea & 0 & 0 & 8 & - & - & 0 \\
\hline
\end{tabular}

Notes. $N_{\mathrm{H}}$ stands for the number of hydrated objects, $N_{\mathrm{U}}$ is the number of uncertain detections, and $N_{\mathrm{T}}$ is the total number of observed primitive objects. Columns 5 and 6 represent, respectively, the mean values for the band center and depth. In the last column, we show the percentage of hydrated objects within the observed family sample.

Morate et al. 2016, 2018), attributed to charge transfer transitions in oxidized iron, indicative of or associated with the presence of aqueous altered minerals on their surfaces (Vilas \& Gaffey 1989; Vilas 1994; Barucci et al. 1998).

In previous publications (Morate et al. 2016, 2018), it has been shown that two families in the inner main belt present signs of hydration within their members: Erigone and Sulamitis. In the case of the families studied in this work, this feature is also present in a significant number of asteroids in three of the four families: Klio, Chaldaea, and Chimaera. We identify the asteroids that show this absorption band, and quantify its position and its depth.

The method used to analyze the presence of the absorption bands is described in Morate et al. (2018). The only difference is that in the present work we decided to be more conservative, increasing our detection threshold when comparing the band depths with the fit residuals up to $3 \sigma$. In Table 2 we show the results for the four families. These results are discussed in the next section.

\section{Discussion}

In Nesvorný et al. (2015) these four families are classified as primitive, based on the SDSS colors available for some of the family members, namely $\mathrm{C}$ (Klio and Chaldea), and CX (Chimaera and Svea). The available visible spectra in the literature for some of the members of these families confirm their "primitivity" (see Table 3). Apart from the asteroids shown in this table, there are no visible spectra in the literature for any other members of the four families.

From the analysis of the spectra of the four parent bodies, (84) $\mathrm{Klio}^{7}$, (313) Chaldaea, (623) Chimaera, and (329) Svea, we expect that Klio, Chaldaea, and Chimaera will show some degree of hydration with moderately red slopes, while Svea will show no signs of aqueous alteration on its members. This prediction is based on the results obtained in Morate et al. (2016, 2018) and de León et al. $(2016,2018)$ : when the parent body shows the $0.7 \mu \mathrm{m}$ absorption band, other family members show it as well, and when the parent body does not present this feature, there are few to no asteroids with signs of hydration (see Fig. 4 for the spectra of the parent bodies). This is confirmed by the results presented in Sect. 3. In the following subsections we discuss the

7 We do not consider (12) Victoria to be the parent body of Klio. Although it is the biggest asteroid in the family, the two available spectra show discrepancies in its composition, indicating that it might be an interloper inside the family (Masiero et al. 2013). 

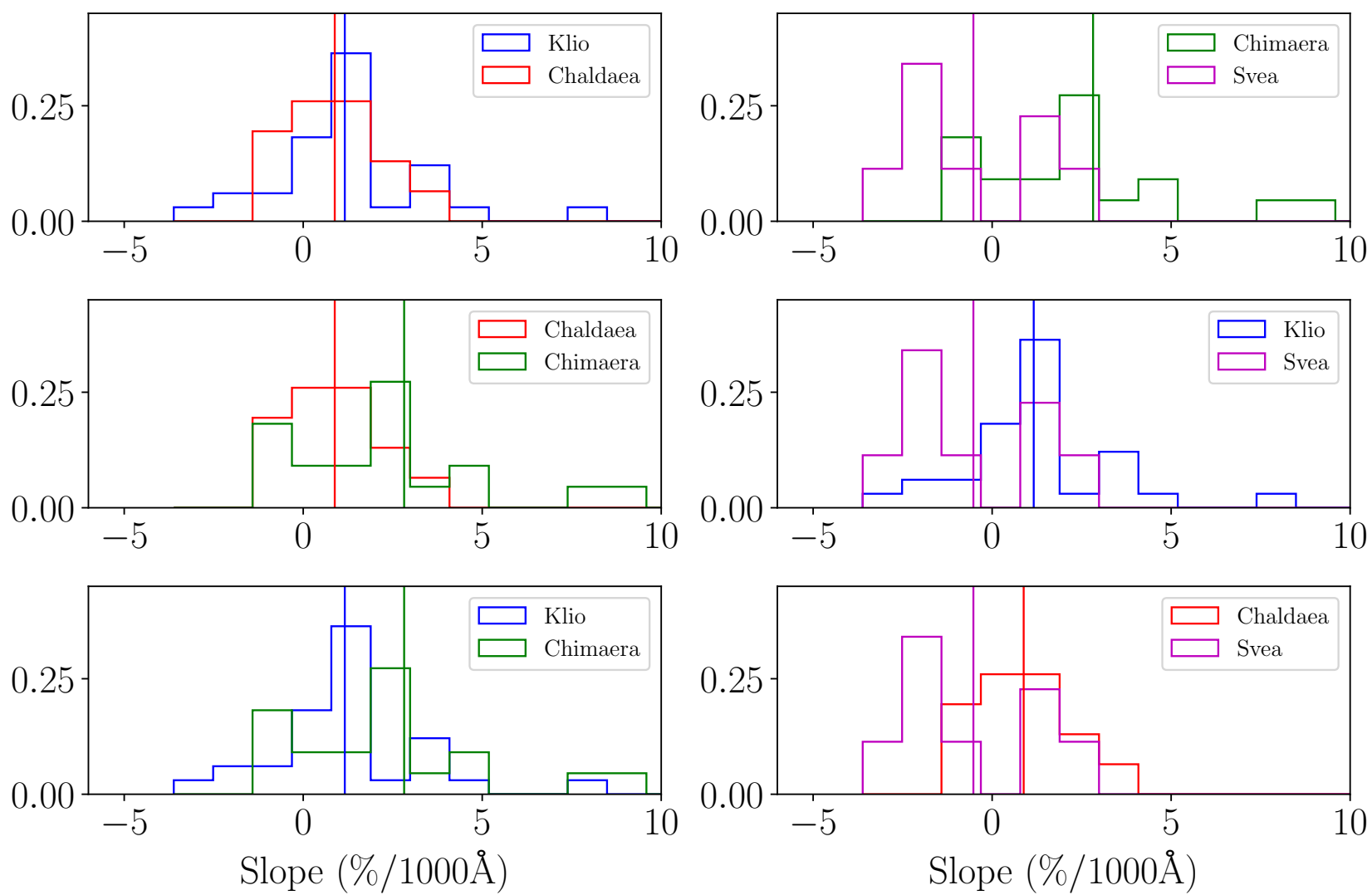

Fig. 3. Normalized histograms comparing the spectral slope distributions of the four families studied in the present work. The bin size is $1.1 \% / 1000 \AA$ A. The Klio family is represented in blue, Chaldaea in red, Chimaera in green, and Svea in magenta. The vertical lines represent the mean values of the slope distributions.

Table 3. Visible spectra of asteroids within the Klio, Chaldaea, and Svea families that are available in the literature, and their classifications.

\begin{tabular}{lcr}
\hline \hline Object & References & Bus Tax. \\
\hline Klio & & \\
\hline 12 & 1,2 & L, D \\
84 & 1,2 & Ch \\
3627 & 1 & B \\
\hline Chaldaea & & \\
\hline 1386 & 1 & Ch \\
5333 & 1 & Ch \\
8106 & 2 & C \\
\hline Svea & & \\
\hline 329 & 2,3 & C \\
\hline
\end{tabular}

Notes. There are no visible spectra in the literature for any of the Chimaera family members.

References. (1) Bus \& Binzel (2002b); (2) Lazzaro et al. (2004); (3) Sawyer (2005).

implications of these results, as well as the possible relationships between the primitive families in the inner main belt.

\subsection{Comparison between all the primitive families in the inner main belt}

Including the four families studied in the present work, there are a total of eight primitive asteroid families located in the

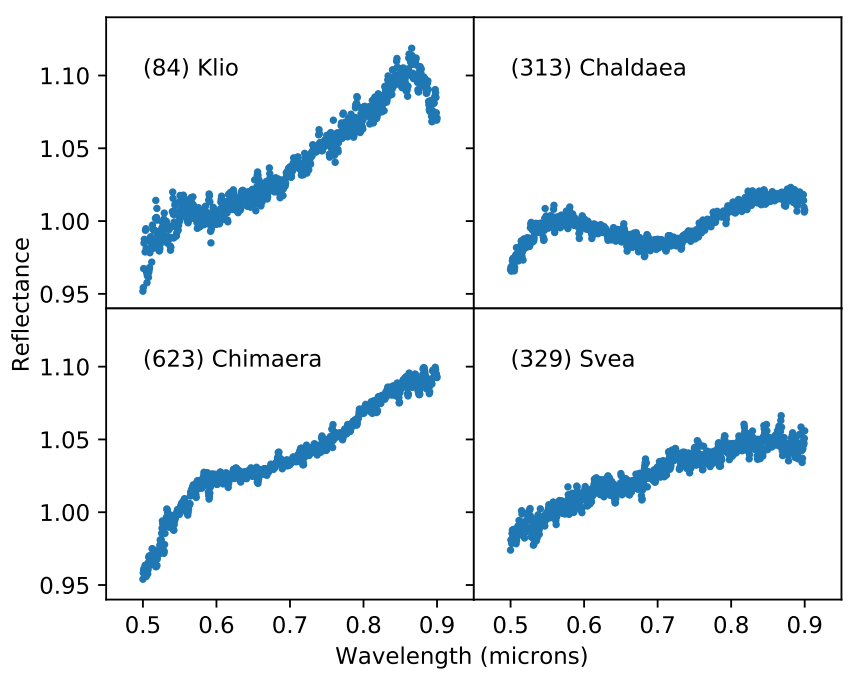

Fig. 4. Spectra of the parent bodies of the four families studied in the present work.

inner main belt. Four of them have already been studied in the visible wavelength range by Morate et al. $(2016,2018)$ and de León et al. (2016, 2018): Polana-Eulalia, Erigone, Clarissa, and Sulamitis. Using the spectral data obtained for these families, de León et al. (2018) proposed that there are two different types of primitive families within the inner main belt: the Polana-like group, whose members lack the $0.7 \mu \mathrm{m}$ absorption band and present homogeneous spectra ranging from slightly blue to moderately red slopes, and the Erigone-like group, whose members show a more extensive spectral diversity and a 
high fraction of them present the $0.7 \mu \mathrm{m}$ feature associated with the presence of phyllosilicates.

The most conspicuous comparison arises from the similarity of the Svea family with the Polana-like group. The sample of the Svea family presented here comprises approximately $17 \%$ of the family, giving a good approximation of its overall composition. None of the observed asteroids shows the $0.7 \mu \mathrm{m}$ feature, one of the main characteristics of the Polana-like families. In addition, the mean spectral slope is $-0.52 \pm 2.04 \% / 1000 \AA$, in good agreement with the spectral slope of the Polana-Eulalia and Clarissa families combined, which is $-0.27 \pm 1.60 \% / 1000 \AA$. This family seems to present a higher fraction of B-type asteroids and a smaller fraction of C-types than its low-inclination counterparts. However, this might be driven by the reduced number of observed asteroids, introducing an observational bias due to the low number of objects.

The other three families studied in this paper fit into the Erigone-like group, although they present some differences. All three of them show neutral to moderately red slopes in their spectra. Klio and Chimaera are spectrally heterogeneous, while Chaldaea are mostly C-types. This result is in contrast with the near-infrared study of Klio by Arredondo et al. (in prep.), where they find that the Klio family is spectrally homogeneous in the near-infrared wavelength range. The three families show hydration to some degree, the percentage in Klio and Chimaera being similar ( $\sim 23$ and $\sim 29 \%$, respectively), yet Chaldaea shows a much stronger presence of hydration $(\sim 79 \%)$. This is a bit different from the fraction of hydrated objects in the Erigone and Sulamitis families (around 50\%). Regarding their slopes, Chimaera shows a redder distribution (mean slope of $2.81 \pm 3.39 \% / 1000 \AA$ ) than those of Klio and Chaldaea, whose mean values are $1.16 \pm 2.04$ and $0.88 \pm 1.25 \% / 1000 \AA$, respectively. However, the mean slope of the combinated distribution of Erigone and Sulamitis asteroids is $1.94 \pm 1.96 \% / 1000 \AA$, which falls in between the values of Klio, Chaldaea, and Chimaera. These three families have both similar slopes to Erigone and Sulamitis and different fraction of hydration traits. Hence, it is difficult to say if they have similar or different origins (see details below).

In order to quantify similarities and differences between the high-inclination families studied in this paper and their low-inclination counterparts, we ran a two-sample KolmogorovSmirnov test $\left(\mathrm{KS}_{\mathrm{S}}\right.$ test $\left.^{8}\right)$, comparing the slope distributions of Klio, Chaldaea, Chimaera, and Svea, with the combinations of the slope distributions of Polana-Eulalia and Clarissa on the one hand, and Erigone and Sulamitis on the other.

To decide whether the compared samples are from different distributions, we computed the Kolmogorov-Smirnov statistic, $D_{m, n}$, which is defined as

$D_{m, n}=\sup \left|F(x)_{1, m}-F(x)_{2, n}\right|$,

where $F(x)_{1, m}$ and $F(x)_{2, n}$ represent the cumulative distribution functions (CDFs) of the two compared distributions. If $D_{m, n}$ is above some critical value $D_{\text {crit }, m, n}$ defined as

$D_{\text {crit }, m, n}=c(\alpha) \sqrt{\frac{m+n}{m n}}$,

where $c(\alpha)=1.36$ for a confidence level of $\alpha=0.05$, and $m$ and $n$ are the sizes of the compared samples, then the KS-test

8 See Chapter 14 in Press et al. (2007) and references therein.

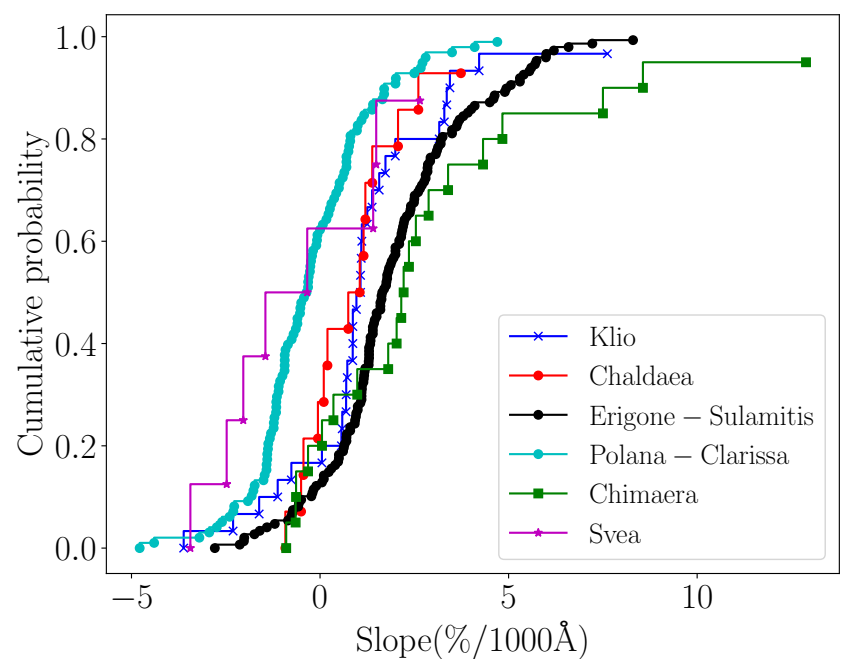

Fig. 5. Cumulative distribution functions of the slope distributions of the four primitive families studied in the present work (Klio, Chaldaea, Chimaera, and Svea), compared to the combined distributions of the Polana and Clarissa families (representing the Polana-like group), and the combined distributions of the Erigone and Sulamitis families (representing the Erigone-like group).

Table 4. Results of the KS-test comparing the Klio, Chaldaea, Chimaera, and Svea families with the distributions representing the Polana-like group (Polana-Eulalia and Clarissa), and the Erigone-like group (Erigone and Sulamitis).

\begin{tabular}{lcccccc}
\hline \hline Family & $D_{\mathrm{PC}}$ & $D_{\mathrm{PC}, \text { crit }}$ & $D_{\mathrm{ES}}$ & $D_{\mathrm{ES}, \text { crit }}$ & Compat $_{\mathrm{PC}}$ & Compat $_{\mathrm{ES}}$ \\
\hline Klio & 0.52 & 0.28 & 0.32 & 0.27 & - & - \\
Chaldaea & 0.40 & 0.38 & 0.36 & 0.38 & - & $\checkmark$ \\
Chimaera & 0.55 & 0.33 & 0.19 & 0.32 & - & $\checkmark$ \\
Svea & 0.34 & 0.50 & 0.52 & 0.49 & $\checkmark$ & - \\
\hline K1 + Ch & 0.45 & 0.24 & 0.32 & 0.23 & - & - \\
\hline
\end{tabular}

Notes. Columns 2 and 3 represent the KS-distance when comparing with the Polana-like distribution, and the critical value of the KS-test in that case, respectively, and Cols. 4 and 5 represent the results of the comparison with the Erigone-like distribution, and the associated critical value. The last two columns show directly the compatibility of the families with the proposed primitive groups.

confirms that the two samples originated in different distributions. A graphical representation of the comparisons made with the KS-test can be seen in Fig. 5, where we show the analyzed cumulative distribution functions.

Data shown in Table 4 indicates that of the four studied families the only one that might be related to the Polana-like group is Svea, confirming what we previously mentioned regarding their taxonomic distribution and the absence of hydration. On the other hand, only Chimaera and Chaldaea show a possible compatibility with the Erigone-like group. Nonetheless, keeping in mind that the two families show different hydration percentages and mean slopes, compared with the Erigone-like group, in addition to the small difference between the KS-statistic and the critical KS-values we should consider the possibility that they are not related to their low-inclination equivalents.

Klio and Chaldaea. The KS-test rejects the compatibility of the Klio family with both the Polana- and the Erigone-like groups. As stated before, the taxonomic distribution and the percentage of objects showing the $0.7 \mu \mathrm{m}$ absorption band differ from the values associated with the Erigone and Sulamitis 


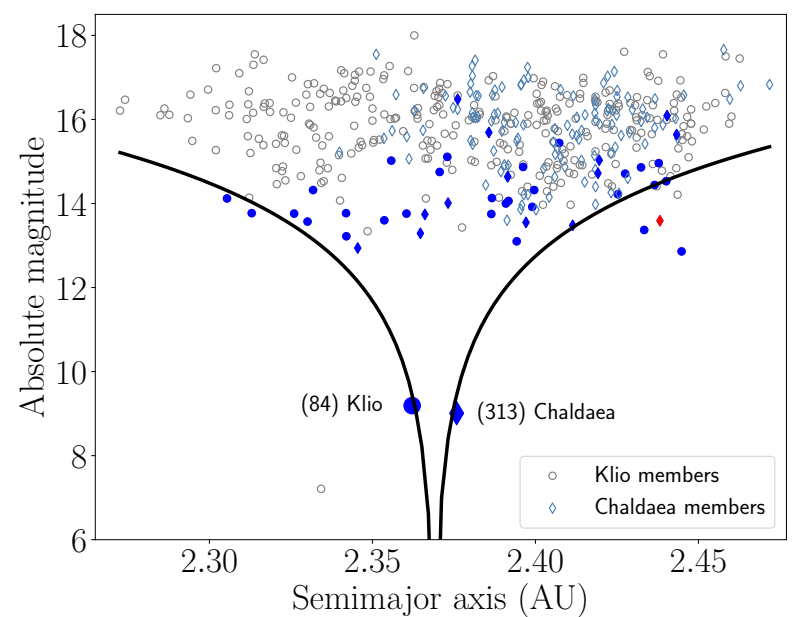

Fig. 6. Absolute magnitude $\left(H_{v}\right)$ of the asteroids from the Klio and Chaldaea families (combined) as a function of their proper semimajor axes. Klio members are plotted as circles, whereas Chaldaea are diamonds. Filled blue symbols correspond to the objects in Klio and Chaldaea observed in this study. The only non-primitive object in the sample is shown in red. The solid black line represents the probable Yarkovsky envelope for the combination of the two families.

families. Interestingly, although Chaldaea is not rejected, their combination (Klio plus Chaldaea) is also considered a different distribution to the Erigone-like group (see Table 4). On the other hand, Klio and Chaldaea both possess partial common traits with the Erigone-like group: Klio shows spectral heterogeneity, and Chaldaea shows a high percentage of hydrated asteroids. However, by themselves, the connection to this primitive group is not clear enough (the fraction of hydrated objects in Klio is lower than expected, and Chaldaea does not show a great spectral variety). However, these two families share common dynamical properties (semimajor axis, eccentricity, and inclination, see Fig. 1), and compositionally they seem to complement each other. If we put together all of the observed asteroids from Klio and Chaldaea into a unique group, this combination yields the following percentages: B-types, 9.1\%, C-types, $65.9 \%$, X-types, $22.7 \%$, and D/T-types, $2.3 \%$, with $\sim 41 \%$ of asteroids showing the $0.7 \mu \mathrm{m}$ band, all values in agreement with what we would expect from an Erigone-like family. This could indicate that the Klio and Chaldaea families might be somehow related.

This hypothesis is further supported by Fig. 6. Here, we can see that the distribution of absolute magnitudes as a function of the semimajor axis for the two families combined shows the typical shape known as the Yarkovsky cone that asteroid families present due to the dynamical spread in semimajor axis experienced by the Yarkovsky thermal forces (Vokrouhlický et al. 2006). The Yarkovsky envelope is computed using the expression

$H=5 \times \log _{10}(\Delta a / C)$,

where $\Delta a=a-a_{\mathrm{c}}$, with $a_{\mathrm{c}}$ defined as the center of the family. In practice, $a_{\mathrm{c}}$ is often close to, or the same as, the semimajor axis of the largest member of the family. In this case $a_{\mathrm{c}}=2.369$, which is the mean value of the semimajor axes of (84) Klio and (313) Chaldaea. The $C$ in Eq. (3) is a constant, which again we computed using the mean values computed for the two families (see Nesvorný et al. 2015), that is, $C=8.75 \times 10^{-5}$ AU. This envelope wraps up the set of the two families as it usually does when only one family is present, leaving the two parental bodies inside it. This is not the case for other family combinations, where both parent bodies fall well outside the Yarkovsky envelope. This, together with the similarity in the orbital elements space, might point to a common origin. Both Chaldaea and Klio might be chunks of a larger, differentiated object having an inner layer of anhydrous silicates covered by a layer of hydrated silicates (see Castillo-Rogez \& McCord 2010, for a model of the interior of Ceres). Chaldaea could be a fragment of the hydrated layer, thus explaining its large fraction of members showing the $0.7 \mu \mathrm{m}$ band, while Klio might be sampling part of the anhydrous layer, showing a small fraction of hydrated objects. The difference in the slope distribution might also be explained this way, where the inner layer, less exposed to space weathering effects, is made of redder material than the outer, hydrated, layer. This would agree with recent laboratory experiments (Lantz et al. 2015, 2017), where they simulate space weathering effects on low-albedo primitive material, suggesting that their visible spectra tend to get bluer as exposure increases.

Despite the previous analysis, we are well aware that this hypothesis needs further study from the dynamical point of view.

\subsection{Klio, Chaldaea, Chimaera, and Svea, as possible sources of (101955) Bennu and (162173) Ryugu}

Near-Earth asteroids (101955) Bennu and (162173) Ryugu are the targets of the sample-return missions OSIRIS-REx and Hayabusa2, respectively. Both missions have arrived at their corresponding targets: Hayabusa2 has already collected surface material from Ryugu, and OSIRIS-REx is orbiting around Bennu, mapping its surface. A summary of the results obtained for the four low-inclination primitive families in the inner main belt regarding their status as possible origins of the two NEAs can be found in de León et al. (2018). We aim to complement those results with data from the smaller primitive families in the inner main belt.

Bennu. There is only one ground-based visible spectrum for (101955) Bennu, from Clark et al. (2011), and one color spectrum from Hergenrother et al. (2013), obtained using ECAS filters. Both datasets, as well as visible and near-infrared spectra obtained with the OVIRS instrument on board OSIRIS-REx, confirm Bennu as a B-type asteroid (Hamilton et al. 2019). Thus, its alleged source family should present also a good fraction of asteroids with a visible B-type spectrum. Among the families studied in the present work, only Svea shows a high enough proportion of B-types ( $\sim 50 \%)$ to be a possible source. However, the family size (48 members) turns Svea into an unlikely origin for asteroid Bennu. Nevertheless, the Svea family lies in the vicinity of both the 3:1 mean motion resonance and the $v_{6}$ secular resonance. In fact, it is the closest blue primitive family to these resonances in the inner belt. Therefore we cannot rule out the possibility of Svea being Bennu's source.

Ryugu. There are several visible spectra of (162173) Ryugu in the literature (Binzel et al. 2001; Vilas 2008; Lazzaro et al. 2013; Moskovitz et al. 2013; Sugita et al. 2013; Perna et al. 2017), all of them classified as $\mathrm{C}$-, $\mathrm{Cb}$-, or Cg-types, with neutral slopes. For this reason, we expect Ryugu to have originated in a family primarily populated by this kind of asteroids. This means that of the four studied families in the present work, Klio, Chaldaea, and Chimaera, might be feasible sources for Ryugu, given that all three show more than $50 \%$ of C-types. However, Chimaera also presents a good fraction of asteroids with moderately red slopes, making it less probable as the source. In addition, the sizes of Klio, Chaldaea, and Chimaera families are relatively small (330, 132 , and 108 members, respectively) compared with the sizes 
of the low-inclination families, which reduces the chances for Ryugu to have its origin within these high-inclination groups.

For both cases, we also have to take into account the inclination of both Bennu and Ryugu, compared to the families studied here. Both asteroids have inclinations $\sin (i) \sim 0.10$, while the mean values for the high-inclination families are all above $\sin (i)>0.15$. According to Bottke et al. (2002b), the $v_{6}$ secular resonance does not have a strong effect on the inclination of the asteroids that enter it. This would favor the low-inclination families as sources of Bennu and Ryugu. However, as we previously stated, the high-inclination families studied in the present work are located very close to the 3:1 and $v_{6}$ resonances, which prevents us from completely discarding them as the sources of the two NEAs.

\subsection{Possible slope-size correlation}

We searched for a possible relationship between the different characteristics of the asteroids within our sample, such as the spectral slope of the primitive objects, the depth and center of the aqueous alteration bands, albedos, sizes, or proper orbital elements. We found no significant correlations between the physical and dynamical properties.

However, when comparing the spectral slope of all the primitive objects belonging to families observed within the inner main belt (low- and high-inclination families) to their computed absolute magnitudes (a sample of 318 asteroids in total), we detected a possible trend in the way the spectral slopes spread depending on the values of $H_{v}$. For larger objects (lower $H_{v}$ values), it seems that the spectral slopes tend to be neutral with relatively little dispersion. As the absolute magnitude increases, this spread tends to increase in a somewhat linear way. We have tried to define a region-of-action for this possible effect (see Fig. 7). If this tendency is real, we would expect the spread in spectral slopes to be roughly contained within the limits of this region, defined by the following empirical formulae for a one-sigma spread:

$H= \begin{cases}-3.414 \times \text { Slope }+10.505 & \text { if Slope }<1.13 \% / 1000 \AA \\ 3.414 \times \text { Slope }+2.774 & \text { if Slope }>1.13 \% / 1000 \AA\end{cases}$

and for a two-sigma spread:

$H= \begin{cases}-1.707 \times \text { Slope }+8.572 & \text { if Slope }<1.13 \% / 1000 \AA \\ 1.707 \times \text { Slope }+4.707 & \text { if Slope }>1.13 \% / 1000 \AA\end{cases}$

These regions are calculated by dividing the absolute magnitude distribution in four equal-sized bins, from $H_{V}=9$ to $H_{V}=19$, computing the mean values of $H_{V}$ inside the bins, and then computing the mean value of the slope distribution, and the corresponding standard deviations of the slopes within the four bins. This will yield eight points (four to each side of the distribution), which we fit to obtain the red and blue straight lines that we see in Fig. 7.

This agrees with the results in Gil-Hutton \& Brunini (2008) and Gil-Hutton \& Licandro (2010), where the authors emphasized that this trend to neutral colors is not seen in the small objects, observed with SDSS. In De Prá et al. (2018a) they find a similar behavior (neutral colors) for the larger asteroids in their analyzed sample.

In that work they propose that larger objects tend to have a saturated surface in terms of environmental effects such as space weathering, which would neutralize the slopes in primitive objects even to the point of becoming negative, as in the B-types (see Vernazza et al. 2013; Lantz et al. 2015, 2017), and collisional resurfacing, which would expose younger and unweathered, thus

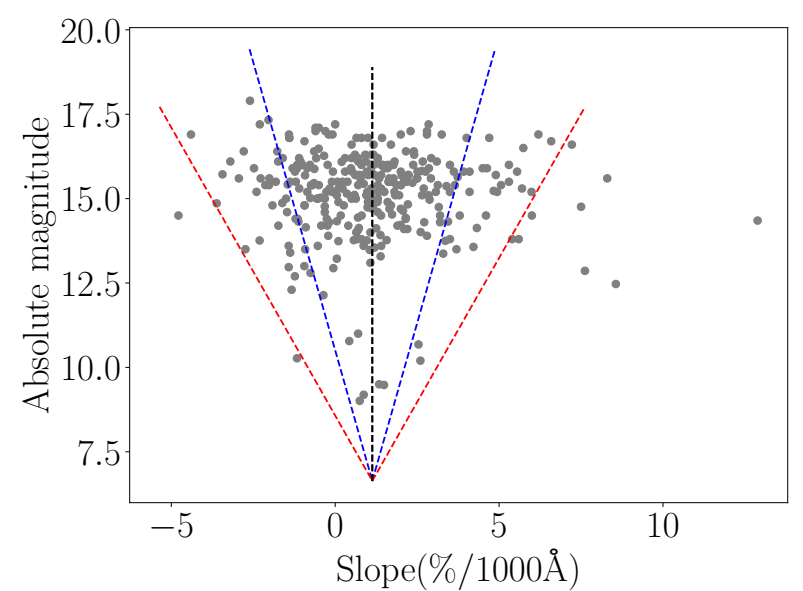

Fig. 7. Distribution of the absolute magnitude of all the observed primitive asteroids within the primitive collisional families located in the inner main belt (Polana, Erigone, Sulamitis, Clarissa, Klio, Chaldaea, Chimaera, and Svea) as a function of the spectral slope. There is a total of 318 observed objects from the eight families. The vertical black line represents the mean value of the slope dispersion, $1.13 \% / 1000 \AA$. The dashed blue and red lines represent the mean spread of the spectral slopes that we might expect (blue for a one-sigma spread, and red for two-sigma). These regions are defined in the main text.

redder, material and which would balance the overall spectral slopes of these bodies (neither positive nor negative).

On the other side, smaller objects could avoid collision for longer periods due to their sizes, presenting old weathered surfaces (blue slopes). However, a collision within a small body might expose a high fraction of fresh material (red slopes). This could influence its spectral slope in a more significant way than in a larger body. Therefore, we suggest that small bodies are more susceptible than larger bodies to slope variations caused by these environmental effects.

We emphasize that this trend is not due to larger uncertainties in the slope since, as explained in Sect. 3.2, the most important source of errors in the slope computation is that associated with the use of different solar analog stars to obtain relative reflectance.

However, we are well aware that this trend might also be produced by an observational bias, due to the lack of data for a high enough number of larger asteroids (there are only 47 asteroids with $H_{v}<14$, and just 9 of them have $H_{v}<11.5$ ). For this reason, we note that it is not an easy task to interpret this behavior, and that this result should be taken into account with caution.

\section{Conclusion and future work}

For the present work we observed and analyzed a total of 73 visible spectra $(0.50-0.90 \mu \mathrm{m})$ of asteroids members of four highinlination families within the inner main belt: Klio, Chaldaea, Chimaera, and Svea. All of them were observed with the $10.4 \mathrm{~m}$ Gran Telescopio Canarias. These four families had never been spectroscopically studied before. The number of objects and sampled percentages of the observed families are as follows: $30 / 330$ in Klio $(\sim 9.1 \%), 15 / 132$ Chaldaea $(\sim 11.3 \%), 20 / 108$ Chimaera $(\sim 18.5 \%)$, and 8/48 Svea $(\sim 16.7 \%)$.

We performed a taxonomical classification of the acquired spectra, which yielded the following results: Klio presents 13.3\% B-types, $56.7 \%$ C-types, 26.7\% X-types, and 3.3\% D/T-types; Chaldaea shows $85.7 \%$ C-types and $14.3 \%$ X-types; in Chimaera, there are 60\% C-types, 20\% X-types, and 20\% D/T-types; finally, in Svea we found $50 \%$ B-types, $37.5 \%$ 
C-types, and $12.5 \%$ X-types. We found only one interloper (an A-type) within the Chaldaea family. The taxonomical distributions of the four families are all different.

Regarding the presence of the $0.7 \mu \mathrm{m}$ band, we found traits of hydration in three of the four families: Klio, Chaldaea, and Chimaera. Out of these three, the family that showed more signs of aqueous alteration is Chaldaea, with approximately $79 \%$, followed by Klio (23\%) and Chimaera (20\%). The eight objects observed from the Svea family showed no absorption bands on their spectra. These numbers are different from those found in previous works for the low-inclination primitive families which showed hydration signs (Erigone and Sulamitis, both around $50 \%$ of hydrated asteroids). This might be related to different evolutionary histories, or simply different hydration levels on the original bodies.

The results we found for Klio and Chaldaea showed that, by themselves, they somehow differ from the Erigone-like families observed to date. However, the properties of their combination resemble those of the Erigone-like families at low inclination. We propose that both families might have a common origin: the Chaldaea family, more hydrated and with bluer slopes, could represent the exterior surface of a primordial object that experienced space weathering effects, while the Klio family might represent the inner layers of the surface of that object, anhydrous and with redder slopes, less affected by space weathering.

We have found that spectral slopes appear to be more "neutral" for larger asteroids, while for smaller objects the slopes tend to be more varied. As in De Prá et al. (2018a), we propose that this effect might be explained by an equilibrium of the influences of space weathering and collisional resurfacing in larger asteroids and a higher variance of the overall surface age in the smaller asteroids population.

We found a clear trend referring primitive family types: to date, all the "blue" families, that is, Polana, Clarissa, and Svea (with slopes ranging from moderately blue to neutral) show no signs of hydration; on the other hand, all the "red" families (Erigone, Sulamitis, Klio, Chaldaea, and Chimaera) present objects with the $0.7 \mu \mathrm{m}$ absorption band, to a greater or lesser extent. Further studies need to be conducted on other primitive families around the main belt in order to confirm whether this trend is happening by chance or if it has a deeper meaning regarding the thermal evolutionary history of the Solar System.

Finally, we compared the four families studied in this work with the spectral properties of (101955) Bennu and (162173) Ryugu that are available in the literature. Of the four families studied in this work, we propose that only Svea is compatible with Bennu, given the high fraction of B-types found in our sample. In the case of Ryugu, Klio, Chaldaea, and Chimaera show compatible spectra with those of the NEA. However, as stated in the previous section, the fact that the sizes of the four families are rather small, together with the differences in the orbit inclinations, shrinks the possibilities of them being the sources of both near-Earth objects.

Acknowledgements. D.M. gratefully acknowledges the Conselho Nacional de Pesquisa (CNPq, Brazil) for the support in the form of a PDJ grant. J.d.L. acknowledges financial support from the Spanish Ministry of Economy and Competitiveness (MINECO) under the 2015 Severo Ochoa Program MINECO SEV2015-0548. M.D.P. acknowledges funding by the Preeminent Postdoctora Program (P3) at the University of Central Florida. J.d.L. and J.L. acknowledge support from the project AYA2015-67772-R (MINECO). N.P.A. gratefully acknowledges support from the Research Opportunities in Space and Earth Sciences-2017 program to the project 17-PDART17 2-0097: PRIMitive Asteroids Spectroscopic Survey (PRIMASS): the past as a puzzle. H.C. acknowledges support from NASA's Near-Earth Object Observations program and the Center for Lunar and Asteroid Surface Science funded by NASA's SSERVI program at the University of Central Florida. J.M.C. acknowledges CPNq for support through a research fellowship. D.L. acknowledges support from CNPq (No. 305409/2016-6 and 409320/2017-0) and FAPERJ/CNE (No. E-26/202.841/2017). The results obtained in this paper are based on observations made with the Gran Telescopio Canarias (GTC), installed in the Spanish Observatorio del Roque de los Muchachos of the Instituto de Astrofísica de Canarias, on the island of La Palma.

\section{References}

Barucci, M. A., Doressoundiram, A., Fulchignoni, M., et al. 1998, Icarus, 132, 388

Bevington, P. R., \& Robinson, D. K. 1992, Data Reduction and Error Analysis for the Physical Sciences (New York: McGraw Hill Education)

Binzel, R. P., Harris, A. W., Bus, S. J., \& Burbine, T. H. 2001, Icarus, 151, 139

Bottke, Jr. W. F., Vokrouhlický, D., Rubincam, D. P., \& Broz, M. 2002a, Asteroids III, eds. W. F. Bottke, Jr. A. Cellino, P. Paolicchi, \& R. P. Binzel (Tucson, AZ: University of Arizona Press) 395

Bottke, W. F., Morbidelli, A., Jedicke, R., et al. 2002b, Icarus, 156, 399

Bottke, W. F., Vokrouhlický, D., Walsh, K. J., et al. 2015, Icarus, 247, 191

Bus, S. J., \& Binzel, R. P. 2002a, Icarus, 158, 146

Bus, S. J., \& Binzel, R. P. 2002b, Icarus, 158, 106

Campins, H., Morbidelli, A., Tsiganis, K., et al. 2010, ApJ, 721, L53

Campins, H., de León, J., Morbidelli, A., et al. 2013, AJ, 146, 26

Carvano, J. M., Mothé-Diniz, T., \& Lazzaro, D. 2003, Icarus, 161, 356

Castillo-Rogez, J. C., \& McCord, T. B. 2010, Icarus, 205, 443

Cepa, J. 2010, in Highlights of Spanish Astrophysics V, eds. J. M. Diego, L. J.

Goicoechea, J. I. González-Serrano, \& J. Gorgas, (Heidelberg: Springer) 15

Cepa, J., Aguiar, M., Escalera, V. G., et al. 2000, SPIE Conf. Ser., 4008, 623

Clark, B. E., Binzel, R. P., Howell, E. S., et al. 2011, Icarus, 216, 462

de León, J., Pinilla-Alonso, N., Delbo, M., et al. 2016, Icarus, 266, 57

de León, J., Campins, H., Morate, D., et al. 2018, Icarus, 313, 25

DeMeo, F. E., Binzel, R. P., Slivan, S. M., \& Bus, S. J. 2009, Icarus, 202, 160

De Prá, M. N., Pinilla-Alonso, N., Carvano, J. M., et al. 2018a, Icarus, 311, 35

De Prá, M. N., Carvano, J., Morate, D., Pinilla-Alonso, N., \& Licandro, J. 2018b,

AAS/Division for Planetary Sciences Meeting Abstracts, 50, 315.02

Fornasier, S., Lazzarin, M., Barbieri, C., \& Barucci, M. A. 1999, A\&AS, 135, 65

Fornasier, S., Lantz, C., Barucci, M. A., \& Lazzarin, M. 2014, Icarus, 233, 163

Gil-Hutton, R., \& Brunini, A. 2008, Icarus, 193, 567

Gil-Hutton, R., \& Licandro, J. 2010, Icarus, 206, 729

Hamilton, V. E., Simon, A. A., Christensen, P. R., et al. 2019, Nat. Astron., 3, 332

Hergenrother, C. W., Nolan, M. C., Binzel, R. P., et al. 2013, Icarus, 226, 663

Howell, E. S., Rivkin, A. S., Vilas, F., et al. 2011, in EPSC-DPS Joint Meeting, 637

Ivezić, Ž., Tabachnik, S., Rafikov, R., et al. 2001, AJ, 122, 2749

Lantz, C., Brunetto, R., Barucci, M. A., et al. 2015, A\&A, 577, A41

Lantz, C., Brunetto, R., Barucci, M. A., et al. 2017, Icarus, 285, 43

Lazzaro, D., Angeli, C. A., Carvano, J. M., et al. 2004, Icarus, 172, 179

Lazzaro, D., Barucci, M. A., Perna, D., et al. 2013, A\&A, 549, L2

Luu, J. X., \& Jewitt, D. C. 1990, AJ, 99, 1985

Masiero, J. R., Mainzer, A. K., Bauer, J. M., et al. 2013, ApJ, 770, 7

Milani, A., Cellino, A., Knežević, Z., et al. 2014, Icarus, 239, 46

Morate, D., de León, J., De Prá, M., et al. 2016, A\&A, 586, A129

Morate, D., de León, J., De Prá, M., et al. 2018, A\&A, 610, A25

Morbidelli, A., Bottke, Jr. W. F., Froeschlé, C., \& Michel, P. 2002, Asteroids III, eds. W. F. Bottke, Jr. A. Cellino, P. Paolicchi, \& R. P. Binzel (Tucson, AZ: University of Arizona Press), 409

Moskovitz, N. A., Abe, S., Pan, K.-S., et al. 2013, Icarus, 224, 24

Nesvorny, D. 2015, NASA Planetary Data System, 234

Nesvorný, D., Brož, M., \& Carruba, V. 2015, Asteroids IV, ed. P. Michel, F. E.

DeMeo, \& W. F. Bottke (Tucson, AZ: University of Arizona Press), 297

Opik, E. J. 1951, Proc. R. Ir. Acad. Sect. A, 54, 165

Perna, D., Barucci, M. A., Ishiguro, M., et al. 2017, A\&A, 599, L1

Pinilla-Alonso, N., de León, J., Walsh, K. J., et al. 2016, Icarus, 274, 231

Press, W. H., Teukolsky, S. A., Vetterling, W. T., \& Flannery, B. P. 2007,

Numerical Recipes: The Art of Scientific Computing, 3rd edn. (New York: Cambridge University Press)

Rivkin, A. S. 2012, Icarus, 221, 744

Sawyer, S. 2005, NASA Planetary Data System, 31, EAR

Sugita, S., Kuroda, D., Kameda, S., et al. 2013, Lunar Planet. Sci. Conf., 44, 2591

Vernazza, P., Fulvio, D., Brunetto, R., et al. 2013, Icarus, 225, 517

Vilas, F. 1994, Icarus, 111, 456

Vilas, F. 2008, AJ, 135, 1101

Vilas, F., \& Gaffey, M. J. 1989, Science, 246, 790

Vokrouhlický, D., Brož, M., Bottke, W. F., Nesvorný, D., \& Morbidelli, A. 2006, Icarus, 182, 118

Zappala, V., \& Cellino, A. 1994, IAU Symp., 160, 395

Zappala, V., Cellino, A., Farinella, P., \& Knezevic, Z. 1990, AJ, 100, 2030 


\section{Appendix A: Visible spectra}

Figures A.1-A.4 show the visible spectra of the asteroids from the four families studied in this paper: Klio, Chimaera, Chaldaea, and Svea. A total of 73 asteroids were observed: 30 from Klio, 20 from Chimaera, 15 from Chaldaea, and 8 from Svea. Spectra are normalized to unity at $0.55 \mu \mathrm{m}$.

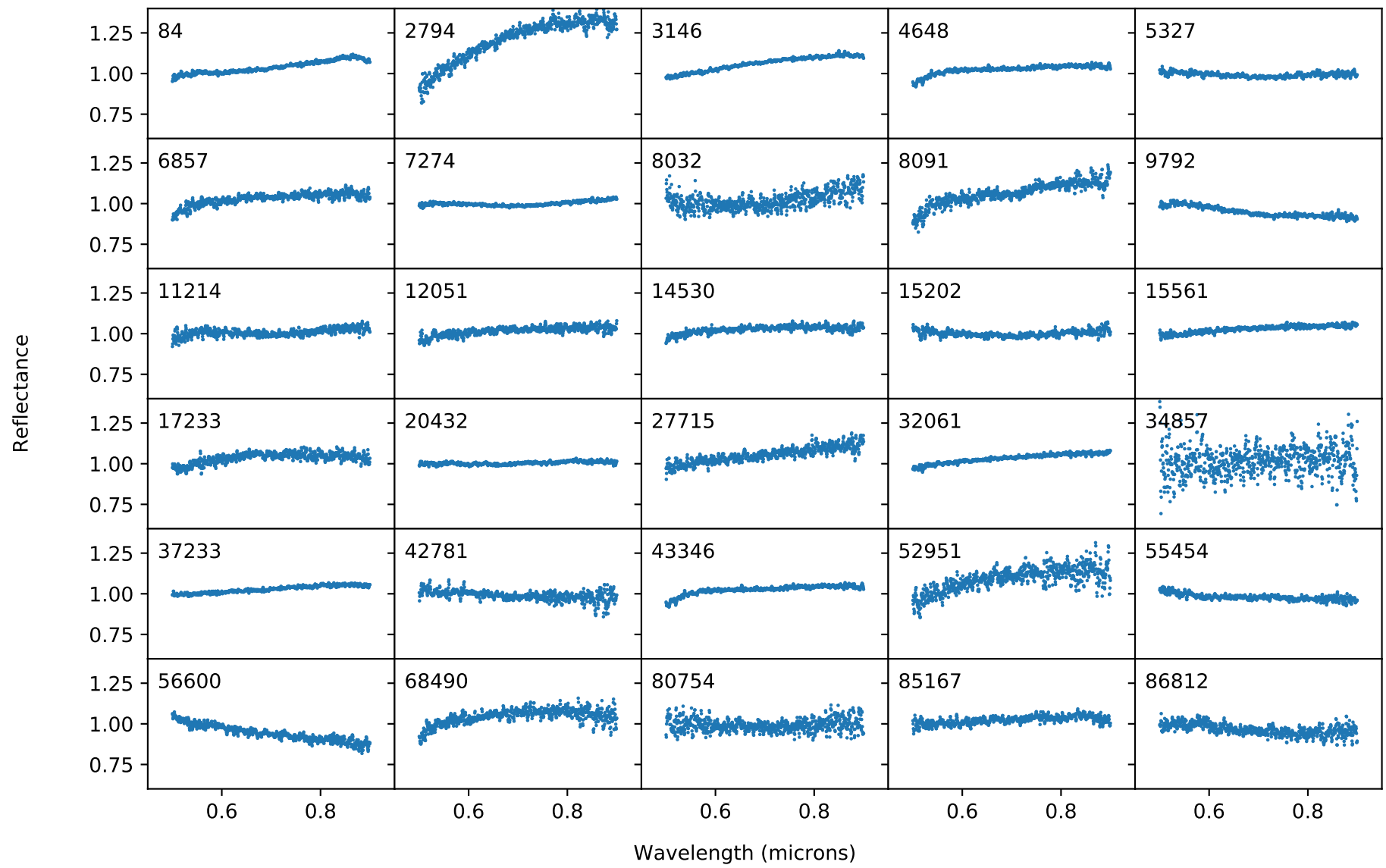

Fig. A.1. Visible spectra of the observed asteroids from the Klio family.

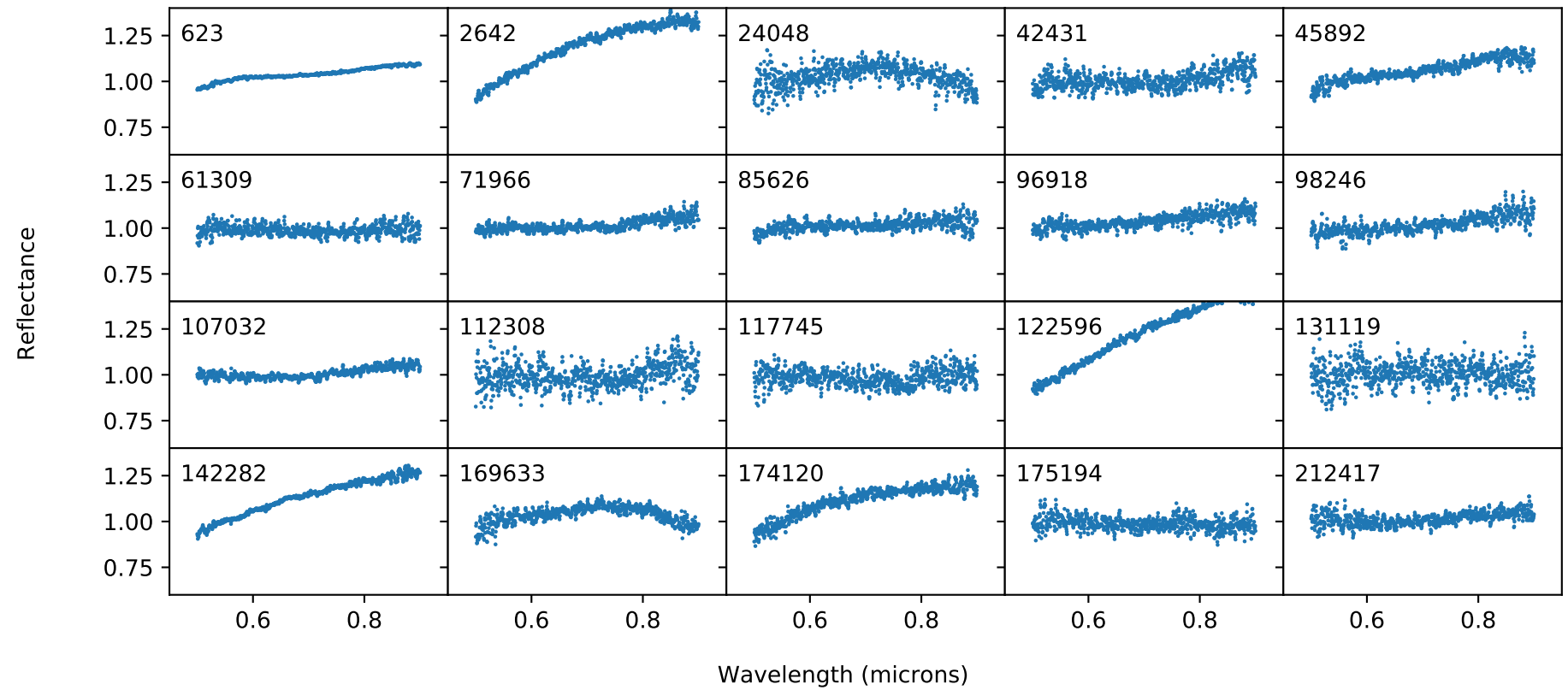

Fig. A.2. Visible spectra of the observed asteroids from the Chimaera family. 
D. Morate et al.: The last pieces of the primitive inner belt puzzle: Klio, Chaldaea, Chimaera, and Svea

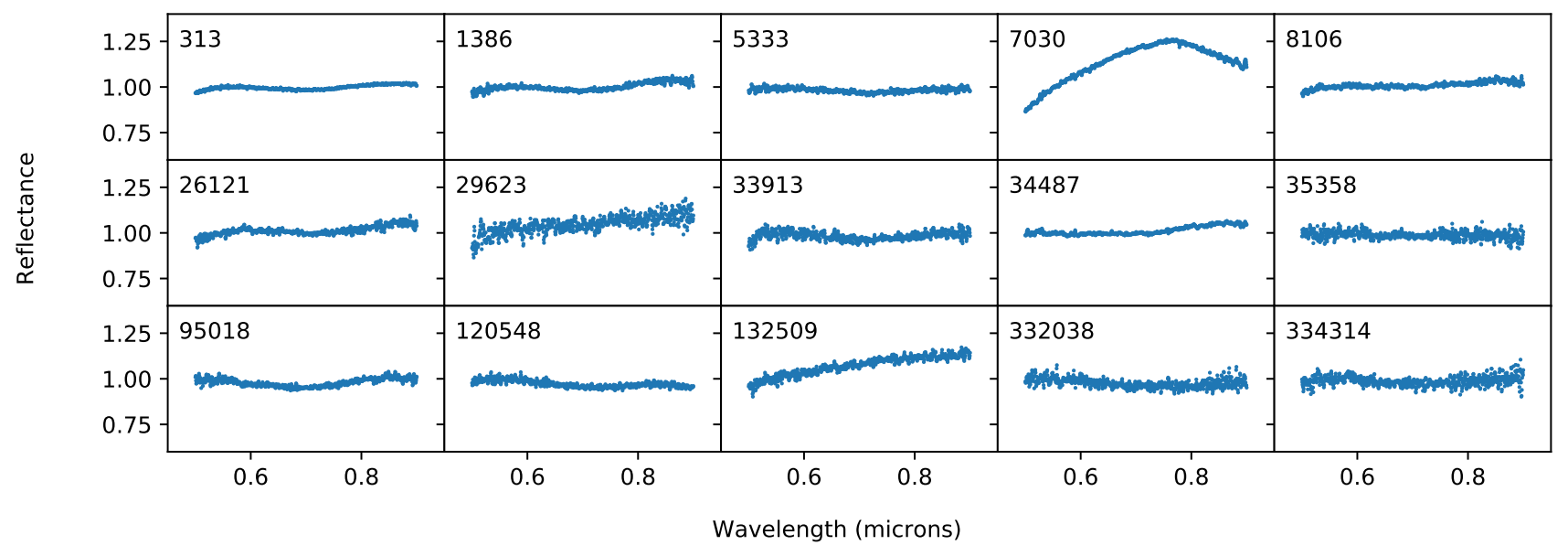

Fig. A.3. Visible spectra of the observed asteroids from the Chaldaea family.

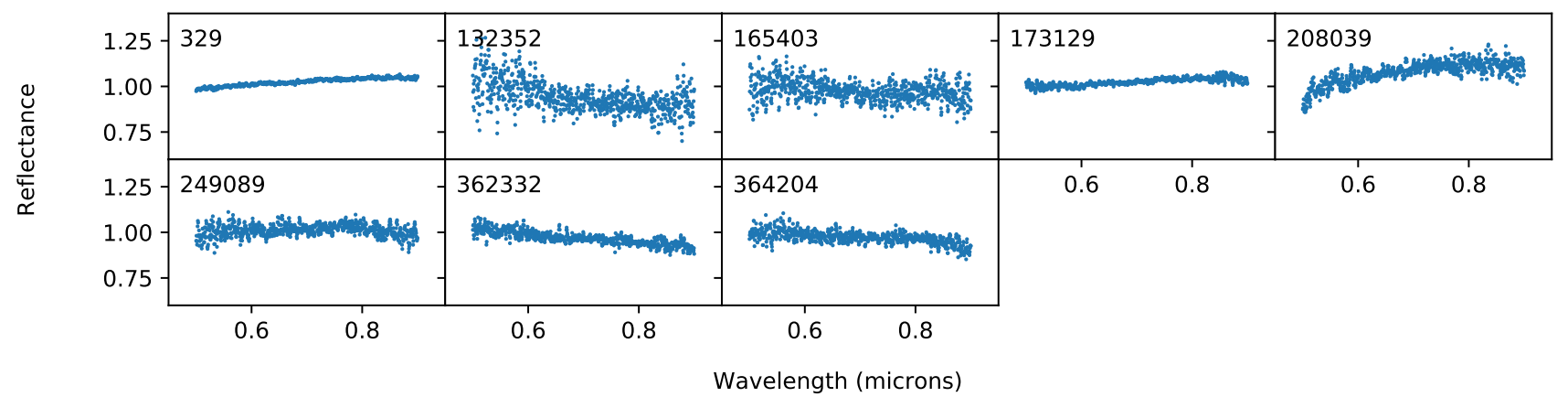

Fig. A.4. Visible spectra of the observed asteroids from the Svea family.

\section{Appendix B: Additional tables}

Table B.1. Observational circumstances for the asteroids presented in this work.

\begin{tabular}{cccccccccc}
\hline \hline Oject & Date & UT Start & $\operatorname{Exp}(\mathrm{s})$ & $m_{v}$ & Phase angle $\left(^{\circ}\right)$ & $\Delta(\mathrm{AU})$ & $r(\mathrm{AU})$ & SAs & Airmass \\
\hline Klio & & & & & & & & & \\
\hline 84 & $2017-02-16$ & $06: 21$ & $3 \times 5$ & 14.4 & 21.8 & 2.37 & 2.652 & 3 & 1.79 \\
2794 & $2016-10-06$ & $21: 12$ & $3 \times 15$ & 17.0 & 25.0 & 1.69 & 2.235 & 5,6 & 1.54 \\
3146 & $2017-02-16$ & $05: 42$ & $3 \times 200$ & 16.9 & 5.0 & 1.56 & 2.539 & 3 & 2.03 \\
4648 & $2016-09-11$ & $00: 22$ & $3 \times 15$ & 16.1 & 4.5 & 1.32 & 2.320 & 7 & 1.1 \\
5327 & $2016-09-25$ & $04: 16$ & $3 \times 300$ & 18.4 & 20.6 & 2.22 & 2.706 & 1 & 1.12 \\
6857 & $2016-10-12$ & $06: 02$ & $2 \times 400$ & 19.3 & 21.5 & 2.65 & 2.702 & 2 & 1.07 \\
7274 & $2017-01-09$ & $00: 46$ & $3 \times 300$ & 17.3 & 9.1 & 1.43 & 2.372 & $1,2,8$ & 1.02 \\
8032 & $2016-09-25$ & $05: 34$ & $3 \times 300$ & 19.3 & 20.9 & 2.61 & 2.805 & 1 & 1.05 \\
8091 & $2016-10-05$ & $05: 35$ & $3 \times 300$ & 18.8 & 23.2 & 2.54 & 2.377 & 6,7 & 1.34 \\
9792 & $2016-09-25$ & $02: 06$ & $3 \times 120$ & 17.3 & 7.3 & 1.45 & 2.428 & 1 & 1.39 \\
11214 & $2017-01-15$ & $05: 12$ & $3 \times 120$ & 17.7 & 27.8 & 1.62 & 2.062 & 2,9 & 1.27 \\
12051 & $2016-09-25$ & $02: 47$ & $3 \times 300$ & 19.0 & 19.4 & 2.09 & 2.698 & 1 & 1.12 \\
14530 & $2016-09-25$ & $03: 31$ & $3 \times 300$ & 18.7 & 21.7 & 1.86 & 2.461 & 1 & 1.06 \\
15202 & $2016-09-25$ & $03: 57$ & $3 \times 120$ & 17.4 & 29.5 & 1.26 & 1.889 & 1 & 1.12 \\
15561 & $2016-09-25$ & $02: 23$ & $3 \times 15$ & 16.0 & 10.7 & 0.89 & 1.868 & 1 & 1.17 \\
17233 & $2016-10-04$ & $23: 09$ & $3 \times 120$ & 18.0 & 24.9 & 1.34 & 2.042 & 6,7 & 1.62 \\
20432 & $2017-02-16$ & $04: 07$ & $3 \times 240$ & 17.8 & 5.8 & 1.74 & 2.712 & 3 & 1.23 \\
27715 & $2017-01-25$ & $19: 49$ & $3 \times 240$ & 18.9 & 19.4 & 1.84 & 2.510 & 8 & 1.06 \\
32061 & $2016-10-11$ & $00: 05$ & $3 \times 240$ & 18.1 & 3.6 & 1.73 & 2.725 & 1,2 & 1.13 \\
\hline
\end{tabular}

Notes. Columns are, from left to right: asteroid number, date of observation, hour of observation (in UT), number of spectra $\times$ exposure time of each spectrum, apparent visual magnitude, phase angle, geocentric distance, heliocentric distance, solar analogs used to obtain the reflectance spectra, and mean airmass of the observation. See Table 1 for the ID number of each solar analogue star. 
Table B.1. continued.

\begin{tabular}{|c|c|c|c|c|c|c|c|c|c|}
\hline Oject & Date & UT Start & $\operatorname{Exp}(s)$ & $m_{v}$ & Phase angle $\left(^{\circ}\right)$ & $\Delta(\mathrm{AU})$ & $r(\mathrm{AU})$ & SAs & Airmass \\
\hline 34857 & 2017-01-08 & 23:00 & $3 \times 360$ & 19.9 & 21.7 & 2.07 & 2.543 & $1,2,8$ & 1.37 \\
\hline 37233 & $2017-02-16$ & 04:30 & $3 \times 240$ & 17.7 & 5.5 & 1.29 & 2.269 & 3 & 1.37 \\
\hline 42781 & 2016-10-04 & $23: 52$ & $3 \times 300$ & 18.9 & 16.4 & 1.82 & 2.610 & 6,7 & 1.42 \\
\hline 43346 & 2017-01-15 & $02: 56$ & $3 \times 240$ & 18.1 & 5.0 & 1.76 & 2.733 & 2,9 & 1.06 \\
\hline 52951 & 2016-11-23 & $21: 22$ & $3 \times 240$ & 19.6 & 20.1 & 1.98 & 2.578 & 1,7 & 1.2 \\
\hline 55454 & $2017-02-25$ & $00: 05$ & $3 \times 240$ & 18.8 & 26.7 & 1.41 & 2.021 & 3 & 1.48 \\
\hline 56600 & $2016-12-29$ & $23: 49$ & $3 \times 300$ & 19.2 & 19.7 & 1.8 & 2.470 & 2 & 1.3 \\
\hline 68490 & 2016-11-23 & $21: 55$ & $3 \times 350$ & 19.7 & 17.8 & 1.7 & 2.465 & 1,7 & 1.2 \\
\hline 80754 & $2016-11-23$ & $20: 53$ & $3 \times 360$ & 20.4 & 18.4 & 2.13 & 2.745 & 1,7 & 1.18 \\
\hline 85167 & $2017-02-16$ & $02: 52$ & $3 \times 120$ & 18.5 & 18.7 & 1.52 & 2.299 & 3 & 1.93 \\
\hline 86812 & 2016-12-29 & 23:00 & $3 \times 450$ & 20.0 & 16.7 & 2.14 & 2.823 & 2 & 1.19 \\
\hline \multicolumn{10}{|l|}{ Chaldaea } \\
\hline 313 & $2016-09-25$ & $02: 36$ & $3 \times 5$ & 12.7 & 17.0 & 1.57 & 2.398 & 1 & 1.14 \\
\hline 1386 & 2016-10-11 & 05:53 & $3 \times 300$ & 18.8 & 20.9 & 2.72 & 2.775 & 1,2 & 1.13 \\
\hline 5333 & 2017-01-15 & $04: 54$ & $3 \times 200$ & 16.5 & 28.2 & 1.37 & 1.952 & 2,9 & 1.35 \\
\hline 7030 & 2016-09-11 & $01: 34$ & $3 \times 200$ & 17.8 & 22.1 & 1.66 & 2.331 & 7 & 1.32 \\
\hline 8106 & 2016-09-11 & 00:47 & $3 \times 250$ & 18.5 & 16.8 & 2.14 & 2.849 & 7 & 1.37 \\
\hline 26121 & 2016-10-06 & $22: 28$ & $3 \times 300$ & 18.2 & 18.5 & 1.88 & 2.585 & 5,6 & 1.26 \\
\hline 29623 & 2016-11-23 & $21: 40$ & $3 \times 60$ & 17.9 & 21.8 & 1.49 & 2.201 & 1,7 & 1.15 \\
\hline 33913 & $2017-01-15$ & $03: 20$ & $3 \times 120$ & 17.1 & 15.8 & 1.29 & 2.165 & 2,9 & 1.2 \\
\hline 34487 & $2017-02-16$ & 04:53 & $3 \times 240$ & 18.0 & 26.2 & 1.25 & 1.940 & 3 & 1.79 \\
\hline 35358 & 2016-11-21 & $20: 18$ & $3 \times 60$ & 17.8 & 22.4 & 1.11 & 1.925 & 7 & 1.07 \\
\hline 95018 & 2017-02-16 & 03:41 & $3 \times 240$ & 18.3 & 10.1 & 1.48 & 2.410 & 3 & 1.45 \\
\hline 120548 & 2017-01-15 & $04: 28$ & $3 \times 240$ & 18.0 & 3.0 & 1.1 & 2.088 & 2,9 & 1.36 \\
\hline 132509 & 2017-01-15 & $03: 53$ & $3 \times 240$ & 18.4 & 9.2 & 1.1 & 2.062 & 2,9 & 1.36 \\
\hline 332038 & $2016-11-23$ & $22: 25$ & $3 \times 120$ & 18.8 & 25.8 & 0.95 & 1.756 & 1,7 & 1.24 \\
\hline 334314 & 2016-11-21 & 20:01 & $3 \times 240$ & 19.1 & 20.3 & 1.18 & 2.009 & 7 & 1.09 \\
\hline \multicolumn{10}{|l|}{ Chimaera } \\
\hline 623 & 2017-08-18 & $05: 26$ & $4 \times 60$ & 14.5 & 13.0 & 1.5 & 2.424 & 7 & 1.35 \\
\hline 2642 & $2017-03-05$ & $06: 26$ & $3 \times 180$ & 17.7 & 21.1 & 2.41 & 2.730 & 2,4 & 1.38 \\
\hline 24048 & 2017-04-12 & 03:11 & $3 \times 500$ & 19.1 & 18.1 & 2.1 & 2.760 & 4 & 1.52 \\
\hline 42431 & 2017-03-04 & 23:09 & $3 \times 450$ & 19.3 & 8.3 & 1.83 & 2.770 & 2,4 & 1.27 \\
\hline 45892 & 2017-03-03 & 03:02 & $3 \times 180$ & 17.6 & 15.7 & 1.38 & 2.250 & 3 & 1.17 \\
\hline 61309 & 2017-03-04 & $21: 23$ & $3 \times 480$ & 19.7 & 17.8 & 2.35 & 2.923 & 2,4 & 1.02 \\
\hline 71966 & 2017-03-03 & $02: 22$ & $3 \times 500$ & 19.0 & 8.3 & 1.95 & 2.885 & 3 & 1.03 \\
\hline 85626 & 2017-03-05 & $05: 50$ & $3 \times 500$ & 19.4 & 18.9 & 1.85 & 2.542 & 2,4 & 1.31 \\
\hline 96918 & 2017-03-03 & 03:18 & $3 \times 500$ & 18.8 & 18.6 & 1.48 & 2.275 & 3 & 1.46 \\
\hline 98246 & 2017-03-03 & $01: 41$ & $3 \times 500$ & 19.0 & 9.9 & 1.46 & 2.400 & 3 & 1.41 \\
\hline 107032 & 2017-03-04 & $20: 57$ & $3 \times 360$ & 19.0 & 18.7 & 1.38 & 2.195 & 2,4 & 1.18 \\
\hline 112308 & 2017-04-05 & $21: 55$ & $3 \times 480$ & 19.8 & 16.6 & 1.74 & 2.536 & 2,3 & 1.01 \\
\hline 117745 & 2017-06-03 & 05:11 & $3 \times 375$ & 19.5 & 30.0 & 1.71 & 2.025 & 5 & 1.14 \\
\hline 122596 & 2017-03-04 & $20: 34$ & $3 \times 300$ & 18.8 & 20.2 & 1.77 & 2.443 & 2,4 & 1.04 \\
\hline 131119 & 2017-05-09 & $00: 39$ & $3 \times 480$ & 19.8 & 14.9 & 2.07 & 2.865 & 4 & 1.89 \\
\hline 142282 & 2017-06-04 & $04: 45$ & $3 \times 400$ & 18.8 & 11.4 & 1.51 & 2.452 & 5 & 1.86 \\
\hline 169633 & 2017-05-07 & 23:01 & $3 \times 500$ & 19.8 & 21.9 & 1.54 & 2.259 & 4 & 1.01 \\
\hline 174120 & $2017-05-08$ & $02: 36$ & $3 \times 500$ & 19.2 & 13.3 & 1.47 & 2.386 & 4 & 1.08 \\
\hline 175194 & 2017-05-08 & 01:01 & $3 \times 500$ & 19.6 & 6.2 & 1.84 & 2.828 & 4 & 1.95 \\
\hline 212417 & 2017-05-08 & $01: 37$ & $3 \times 500$ & 18.2 & 5.3 & 1.12 & 2.126 & 4 & 1.86 \\
\hline \multicolumn{10}{|l|}{ Svea } \\
\hline 329 & 2017-03-05 & $06: 41$ & $3 \times 10$ & 14.0 & 22.4 & 1.86 & 2.419 & 2,4 & 1.27 \\
\hline 173129 & 2017-04-01 & $02: 25$ & $3 \times 480$ & 19.2 & 10.4 & 1.61 & 2.543 & 3 & 1.19 \\
\hline 208039 & 2017-03-31 & 23:01 & $3 \times 480$ & 20.1 & 13.6 & 1.6 & 2.486 & 3 & 1.19 \\
\hline 132352 & 2017-04-05 & $23: 24$ & $3 \times 420$ & 20.2 & 14.8 & 1.73 & 2.574 & 2,3 & 1.02 \\
\hline 165403 & 2017-04-05 & $22: 38$ & $3 \times 480$ & 20.4 & 19.8 & 1.94 & 2.579 & 2,3 & 1.02 \\
\hline 249089 & 2017-04-06 & 00:18 & $3 \times 480$ & 19.8 & 8.0 & 1.43 & 2.401 & 2,3 & 1.35 \\
\hline 362332 & $2017-05-07$ & $23: 48$ & $3 \times 500$ & 19.7 & 3.2 & 1.63 & 2.642 & 4 & 1.37 \\
\hline 364204 & $2017-05-08$ & $00: 24$ & $3 \times 500$ & 19.5 & 5.5 & 1.06 & 2.060 & 4 & 1.3 \\
\hline
\end{tabular}


Table B.2. Results obtained from the analyses performed in the present work.

\begin{tabular}{|c|c|c|c|c|c|c|}
\hline Object & Class & $\begin{array}{c}\text { Slope } \\
( \pm 0.9 \% / 1000 \AA)\end{array}$ & $H_{V}$ & Band & $\begin{array}{l}\text { Depth } \\
(\%)\end{array}$ & $\begin{array}{l}\text { Center } \\
(\AA)\end{array}$ \\
\hline Klio & & $1.16 \pm 2.04$ & & & & \\
\hline 84 & $X$ & 0.87 & 9.2 & $\checkmark$ & $1.60 \pm 0.03$ & $6880 \pm 10$ \\
\hline 2794 & $\mathrm{D}$ & 7.62 & 12.9 & - & - & - \\
\hline 3146 & $\mathrm{X}$ & 3.30 & 13.4 & - & - & - \\
\hline 4648 & $\mathrm{Cb}$ & 1.07 & 13.1 & - & - & - \\
\hline 5327 & $\mathrm{Ch}$ & 0.05 & 13.2 & $\checkmark$ & $2.39 \pm 0.04$ & $6995 \pm 15$ \\
\hline 6857 & $\mathrm{Cb}$ & 1.57 & 13.8 & - & - & - \\
\hline 7274 & $\mathrm{Cgh}$ & 0.96 & 13.8 & $\checkmark$ & $2.55 \pm 0.02$ & $7138 \pm 8$ \\
\hline 8032 & $\mathrm{Xc}$ & 3.36 & 13.8 & UNC & $3.91 \pm 0.16$ & $6810 \pm 50$ \\
\hline 8091 & $X$ & 4.22 & 13.6 & - & - & - \\
\hline 9792 & B & -2.31 & 13.8 & $\checkmark$ & $2.84 \pm 0.04$ & $7112 \pm 8$ \\
\hline 11214 & $\mathrm{Cgh}$ & 0.87 & 13.6 & $\checkmark$ & $2.52 \pm 0.07$ & $7176 \pm 12$ \\
\hline 12051 & $\mathrm{Cb}$ & 1.25 & 14.1 & - & - & - \\
\hline 14530 & $\mathrm{Cb}$ & 0.72 & 14.1 & - & - & - \\
\hline 15202 & $\mathrm{Cgh}$ & 0.69 & 14.1 & $\checkmark$ & $2.38 \pm 0.06$ & $7000 \pm 30$ \\
\hline 15561 & $\mathrm{Cb}$ & 1.38 & 13.9 & - & - & - \\
\hline 17233 & $\mathrm{Cb}$ & 0.69 & 14.4 & - & - & - \\
\hline 20432 & $\mathrm{Cb}$ & 0.57 & 13.8 & $\checkmark$ & $0.97 \pm 0.03$ & $6710 \pm 20$ \\
\hline 27715 & $X$ & 3.44 & 14.3 & - & - & - \\
\hline 32061 & $\mathrm{Xk}$ & 2.00 & 14.2 & - & - & - \\
\hline 34857 & $\mathrm{Cb}$ & 1.09 & 15.0 & - & - & - \\
\hline 37233 & $\mathrm{Cb}$ & 1.73 & 14.5 & - & - & - \\
\hline 42781 & $\mathrm{~B}$ & -1.12 & 14.3 & - & - & - \\
\hline 43346 & $\mathrm{Cb}$ & 1.07 & 14.0 & - & - & - \\
\hline 52951 & $\mathrm{Xe}$ & 3.16 & 14.8 & - & - & - \\
\hline 55454 & $\mathrm{Ch}$ & -0.76 & 15.0 & - & - & - \\
\hline 56600 & $\mathrm{~B}$ & -3.62 & 14.9 & - & - & - \\
\hline 68490 & $\mathrm{Xk}$ & 0.87 & 15.4 & - & - & - \\
\hline 80754 & $\mathrm{Ch}$ & 0.59 & 15.1 & - & - & - \\
\hline 85167 & $\mathrm{Cb}$ & 1.11 & 14.7 & - & - & - \\
\hline 86812 & $\mathrm{~B}$ & -1.61 & 14.9 & UNC & $2.62 \pm 0.15$ & $7330 \pm 30$ \\
\hline Chaldaea & & $0.88 \pm 1.25$ & & & & \\
\hline 313 & $\mathrm{Cgh}$ & 0.75 & 9.0 & $\checkmark$ & $2.40 \pm 0.02$ & $7094 \pm 3$ \\
\hline 1386 & $\mathrm{Cgh}$ & 1.38 & 13.3 & $\checkmark$ & $3.67 \pm 0.03$ & $7089 \pm 6$ \\
\hline 5333 & $\mathrm{Ch}$ & -0.06 & 12.9 & $\checkmark$ & $2.23 \pm 0.04$ & $7140 \pm 10$ \\
\hline 7030 & $\mathrm{~A}$ & - & 13.6 & - & - & - \\
\hline 8106 & $\mathrm{Cb}$ & 1.05 & 13.5 & $\checkmark$ & $1.67 \pm 0.03$ & $7117 \pm 8$ \\
\hline 26121 & $\mathrm{Cgh}$ & 1.20 & 13.6 & $\checkmark$ & $3.17 \pm 0.05$ & $7299 \pm 12$ \\
\hline 29623 & $\mathrm{Xc}$ & 2.61 & 14.0 & - & - & - \\
\hline 33913 & $\mathrm{Ch}$ & 0.10 & 13.7 & $\checkmark$ & $3.88 \pm 0.07$ & $7176 \pm 11$ \\
\hline 34487 & $\mathrm{Cb}$ & 2.07 & 14.6 & $\checkmark$ & $2.99 \pm 0.03$ & $6870 \pm 10$ \\
\hline 35358 & $\mathrm{Ch}$ & -0.44 & 15.0 & - & - & - \\
\hline 95018 & $\mathrm{Ch}$ & 1.16 & 14.7 & $\checkmark$ & $4.94 \pm 0.05$ & $6901 \pm 7$ \\
\hline 120548 & $\mathrm{Ch}$ & -0.92 & 15.7 & $\checkmark$ & $2.95 \pm 0.05$ & $7043 \pm 6$ \\
\hline 132509 & $X$ & 3.74 & 15.6 & - & - & - \\
\hline 332038 & $\mathrm{Ch}$ & -0.50 & 16.5 & $\checkmark$ & $2.96 \pm 0.09$ & $7260 \pm 20$ \\
\hline 334314 & $\mathrm{Ch}$ & 0.20 & 16.1 & $\checkmark$ & $3.28 \pm 0.12$ & $7230 \pm 30$ \\
\hline Chimaera & & $2.82 \pm 3.39$ & & & & \\
\hline 623 & $\mathrm{Xc}$ & 2.54 & 10.7 & $\checkmark$ & $1.32 \pm 0.02$ & $7231 \pm 5$ \\
\hline 2642 & $\mathrm{D}$ & 8.56 & 12.5 & - & - & - \\
\hline 24048 & $\mathrm{Cg}$ & -0.90 & 14.2 & - & - & - \\
\hline 42431 & $\mathrm{Cb}$ & 2.22 & 14.8 & $\checkmark$ & $4.74 \pm 0.19$ & $7210 \pm 30$ \\
\hline 45892 & $\mathrm{X}$ & 4.32 & 14.1 & UNC & $1.74 \pm 0.08$ & $7011 \pm 11$ \\
\hline
\end{tabular}

Notes. We include here the taxonomical classification assigned to each observed object, based on the Bus taxonomy (Bus \& Binzel 2002a), as well as the spectral slopes for the primitive objects, and the aqueous alteration information for those objects showing the $0.7 \mu \mathrm{m}$ absorption band. 
A\&A 630, A141 (2019)

Table B.2. continued.

\begin{tabular}{ccccccc}
\hline \hline Object & Class & $\begin{array}{c}\text { Slope } \\
( \pm 0.9 \% / 1000 \AA)\end{array}$ & $H_{V}$ & Band & $\begin{array}{c}\text { Depth } \\
(\%)\end{array}$ & $\begin{array}{c}\text { Center } \\
(\AA)\end{array}$ \\
\hline 61309 & $\mathrm{Ch}$ & 0.05 & 14.3 & - & - & - \\
71966 & $\mathrm{Cb}$ & 2.15 & 14.4 & $\checkmark$ & $2.79 \pm 0.09$ & $7100 \pm 30$ \\
85626 & $\mathrm{Cb}$ & 0.99 & 14.7 & - & - & - \\
96918 & $\mathrm{Xc}$ & 2.88 & 15.0 & - & - & - \\
98246 & $\mathrm{X}$ & 3.40 & 15.4 & - & - & - \\
107032 & $\mathrm{Ch}$ & 2.36 & 15.5 & $\checkmark$ & $2.87 \pm 0.06$ & $6850 \pm 20$ \\
112308 & $\mathrm{Cgh}$ & 1.81 & 15.2 & $\mathrm{UNC}$ & $5.5 \pm 0.3$ & $7090 \pm 20$ \\
117745 & $\mathrm{Ch}$ & 0.35 & 15.2 & $\mathrm{UNC}$ & $4.3 \pm 0.2$ & $7200 \pm 30$ \\
122596 & $\mathrm{D}$ & 12.89 & 14.3 & - & - & - \\
131119 & $\mathrm{C}$ & -0.32 & 14.8 & - & - & - \\
142282 & $\mathrm{D}$ & 7.50 & 14.8 & - & - & - \\
169633 & $\mathrm{Cg}$ & -0.64 & 15.9 & - & - & - \\
174120 & $\mathrm{~T}$ & 4.84 & 15.2 & - & - & - \\
175194 & $\mathrm{Ch}$ & -0.65 & 15.5 & - & - & - \\
212417 & $\mathrm{Cb}$ & 2.03 & 15.7 & $\mathrm{UNC}$ & $2.70 \pm 0.12$ & $6700 \pm 40$ \\
\hline Svea & & $-0.52 \pm 2.04$ & & & & \\
\hline 329 & $\mathrm{C}$ & 1.49 & 9.5 & - & - & - \\
132352 & $\mathrm{~B}$ & -3.44 & 15.7 & - & - & - \\
165403 & $\mathrm{~B}$ & -1.45 & 15.4 & - & - & - \\
173129 & $\mathrm{Cb}$ & 1.41 & 15.3 & - & - & - \\
208039 & $\mathrm{Xe}$ & 2.65 & 15.8 & - & - & - \\
249089 & $\mathrm{C}$ & -0.34 & 16.3 & - & - & - \\
362332 & $\mathrm{~B}$ & -2.48 & 15.9 & - & - & - \\
364204 & $\mathrm{~B}$ & -2.04 & 17.3 & - & - & - \\
\hline
\end{tabular}

